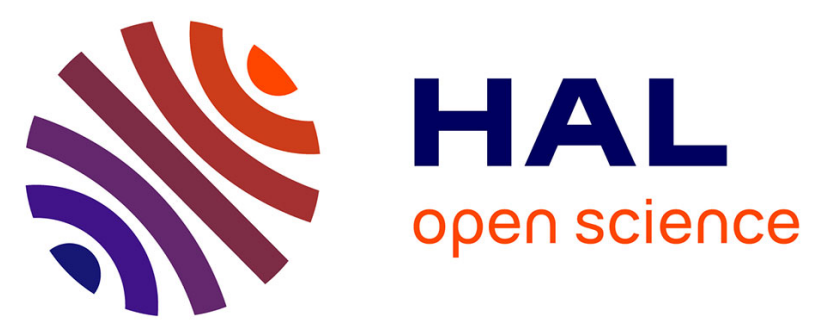

\title{
Can carbon-13 in large herbivores reflect the canopy effect in temperate and boreal ecosystems? Evidence from modern and ancient ungulates
}

Dorothée G. Drucker, Anne Bridault, Keith A Hobson, Elwira Szuma, Hervé Bocherens

\section{To cite this version:}

Dorothée G. Drucker, Anne Bridault, Keith A Hobson, Elwira Szuma, Hervé Bocherens. Can carbon13 in large herbivores reflect the canopy effect in temperate and boreal ecosystems? Evidence from modern and ancient ungulates. Palaeogeography, Palaeoclimatology, Palaeoecology, 2008, 266 (1-2), pp.69-82. 10.1016/j.palaeo.2008.03.020 . hal-02335420

\section{HAL Id: hal-02335420 \\ https://hal.parisnanterre.fr/hal-02335420}

Submitted on 28 Oct 2019

HAL is a multi-disciplinary open access archive for the deposit and dissemination of scientific research documents, whether they are published or not. The documents may come from teaching and research institutions in France or abroad, or from public or private research centers.
L'archive ouverte pluridisciplinaire HAL, est destinée au dépôt et à la diffusion de documents scientifiques de niveau recherche, publiés ou non, émanant des établissements d'enseignement et de recherche français ou étrangers, des laboratoires publics ou privés. 


\title{
Can carbon-13 in large herbivores reflect the canopy effect in temperate and boreal ecosystems? Evidence from modern and ancient ungulates
}

\author{
Dorothée G. Drucker ${ }^{\mathrm{a}, \mathrm{b}, *}$, Anne Bridault ${ }^{\mathrm{c}}$, Keith A. Hobson ${ }^{\mathrm{d}}$, Elwira Szuma ${ }^{\mathrm{e}}$, Hervé Bocherens ${ }^{\mathrm{a}, \mathrm{f}}$ \\ a Prairie and Northern Wildlife Research Centre, Canadian Wildlife Service, Environment Canada, 115 Perimeter Road, Saskatoon, SK, Canada S7N 0X4 \\ b Institut für Ur- und Frühgeschichte und Archäologie des Mittelalters, Naturwissenschaftliche Archäologie, Universität Tübingen, Rümelinstr. 23, D-72070 Tübingen Germany

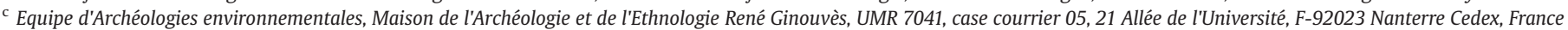 \\ d Environment Canada, 11 Innovation Blvd, Saskatoon, SK, Canada S7N 3H5 \\ e Mammal Research Institute, Polish Academy of Sciences, ul. A. Waszkiewicza 1, 17-230 Bialowieza, Poland \\ ${ }^{\mathrm{f}}$ Institut für Geowissenschaften, Biogeologie, Universität Tübingen, Sigwartstr. 10, D-72076 Tübingen, Germany
}

Keywords:

Canopy effect

Carbon isotopes

Late-Glacial/Early Holocene transition

Large herbivores

\begin{abstract}
A B S T R A C T
Local environmental conditions under dense canopy are known to result in depletion in ${ }^{13} \mathrm{C}$ abundance in plants compared to an open land context. This canopy effect has been observed in tropical as well as in midlatitude forest ecosystems. However, the impact of the canopy effect on tissue ${ }^{13} \mathrm{C}$ abundance of temperate and boreal forest-dwelling herbivores has not been thoroughly explored. Nevertheless, the canopy effect has been suggested to explain a decrease of about $3 \%$ in collagen $\delta^{13} \mathrm{C}$ values in ancient large herbivores from western Europe during the forest expansion of the Late-Glacial-Early Holocene period (ca. 15,000-6000 cal $\mathrm{BP})$. Some papers have considered the ${ }^{13} \mathrm{C}$ decrease in large herbivore as the main result of global change in atmospheric $\mathrm{CO}_{2}$ content. A detailed review of $\delta^{13} \mathrm{C}$ values of large herbivores (reindeer, red deer, roe deer, and bison) from open and closed environments from high and mid-latitudes confirm that the canopy effect observed in plants is passed on to their consumers. In the Paris Basin, the decline in $\delta^{13} \mathrm{C}$ values of large herbivores at the Late-Glacial/Early Holocene transition around 10,000 years BP appears to be different according to the considered species, namely red deer, roe deer, and large bovines (bison and aurochs). Moreover, differences in the pattern of decrease in $\delta^{13} \mathrm{C}$ values are observed in red deer between French northern Alps and French Jura. These differences among species in their isotopic response through time for a given geographical location, and within species from different locations, suggest variance in ecological responses of species that are associated with the relative use of forested habitat. As a result, ${ }^{13} \mathrm{C}$ abundances in collagen can be considered as a direct tracker of the degree of closure of the habitat of ancient herbivores.
\end{abstract}

\section{Introduction}

Habitat use and dietary preferences of ancient herbivores is an important question in palaeobiology. In tropical contexts, the use of carbon isotopic signatures $\left(\delta^{13} \mathrm{C}\right)$ of modern and fossil herbivore tissues is nowadays routinely used to document these issues, since herbaceous plants in open environments and herbaceous and arboreal plants from forested contexts use different types of photosynthesis, $C_{4}$ versus $C_{3}$, which exhibit very distinctive carbon isotopic signatures (e.g. Ambrose and DeNiro, 1986; van der Merwe et al., 1990; Vogel et al., 1990; Cerling et al., 1998). In boreal and temperate ecosystems, where $C_{4}$ plants are practically absent, $C_{3}$ plants exhibit isotopic contrasts according to environmental conditions (see reviews in Tieszen, 1991; Heaton, 1999; Dawson et al., 2002). Variation in $\delta^{13} \mathrm{C}$

* Corresponding author. Institut für Ur- und Frühgeschichte und Archäologie des Mittelalters, Naturwissenschaftliche Archäologie, Universität Tübingen, Rümelinstr. 23, D-72070 Tübingen. Tel.: +49 (0)7071 2976519.

E-mail address: dorothee.drucker@ifu.uni-tuebingen.de (D.G. Drucker). values in $C_{3}$ plants has been observed in response to soil moisture, low humidity, irradiance, temperature, nitrogen availability, salinity, and atmospheric $\mathrm{CO}_{2}$ concentration. Despite these numerous factors influencing ${ }^{13} \mathrm{C}$ assimilation by $\mathrm{C}_{3}$-plants, some systematic patterns are observed between different plant assemblages. One of those patterns is the so called "canopy effect" that corresponds to a vertical gradient in the $\delta^{13} \mathrm{C}$ values of forest trees, with high $\delta^{13} \mathrm{C}$ values at the top of the canopy and low values at the bottom, and to a ${ }^{13} \mathrm{C}$ depletion in plants growing under forested closed canopy relative to the same plant type subject to more open growing conditions (e.g., reviews in Broadmeadow and Griffiths, 1993; Heaton, 1999). This ${ }^{13} \mathrm{C}$ depletion in plants growing under forested closed canopies may be linked to the combination of two factors: (1) atmospheric $\mathrm{CO}_{2}$ available to plants in poorly ventilated understory is ${ }^{13} \mathrm{C}$ depleted relative to the general atmosphere as the result of $\mathrm{CO}_{2}$ recycling from leaf litter (e.g. Schleser and Jayasekera, 1985; Gebauer and Schulze, 1991; van der Merwe and Medina, 1991), (2) a $\mathrm{CO}_{2}$ concentration gradient and light attenuation under the forest canopy leads to depleted ${ }^{13} \mathrm{C}$ abundances in understory plants due to change in photosynthetic activity and 
stomatal conductance (e.g. Francey et al., 1985; Ehleringer et al., 1986; Gebauer and Schulze, 1991; Broadmeadow et al., 1992). Some authors also invoke a higher water availability for plants growing under canopies relative to plants growing in more exposed sites (Broadmeadow et al., 1992; Brooks et al., 1997). Consequently, the intensity of canopy effect is expected to depend on the characteristics of the canopy. General trends are that the more complex and denser the canopy, the greater the extent of light reduction and the degree of recycling of respired forest floor $\mathrm{CO}_{2}$ within the understory layers (France, 1996). For a significant ${ }^{13} \mathrm{C}$ depletion to be observed in arboreal plant formations, two conditions need to be fulfilled: $\mathrm{CO}_{2}$ produced by plant respiration is confined and the gradient of $\mathrm{CO}_{2}$ is not dissipated by wind turbulence (e.g., Broadmeadow and Griffiths, 1993; France, 1996; Roche, 1999), and the light attenuation is sufficient thanks to a high Leaf Area Index (Broadmeadow and Griffiths, 1993; Buchmann et al., 1997).

The "canopy effect" causing changes in ${ }^{13} \mathrm{C}$ abundances of plants is expected to be passed on to herbivores feeding on understory vegetation. Indeed, some studies tentatively established links between ${ }^{13} \mathrm{C}$ abundances in animals and the canopy effect. For instance, depleted ${ }^{13} \mathrm{C}$ abundance has been shown in tissues from elephants living in tropical forest compared to those living in savannah (van der Merwe et al., 1990; Vogel et al., 1990), but the occurrence of $C_{4}$ plants with $\delta^{13} \mathrm{C}$ values as high as $-12 \%$ in savanna grass may contribute to an exaggeration of the difference between the $\delta^{13} \mathrm{C}$ values of both populations. Also, the more negative $\delta^{13} \mathrm{C}$ values measured in South American monkeys living in closed canopy forest compared to those living in more open environments presented by Schoeninger et al. (1997) may be partly due to differences in dietary specialisation between the considered species. One recent attempt to document canopy effect in red deer collagen $\delta^{13} \mathrm{C}$ values based on the comparative study of five European populations from $C_{3}$ contexts remains unconvincing (Stevens et al., 2006), but this outcome is more due to the difficulty of finding red deer populations unaffected by anthropogenic interferences rather than on the absence of the canopy effect (see discussion in the present paper). Finally, there is no ideal example showing a significant difference in $\delta^{13} \mathrm{C}$ values for two populations of the same species, one living under a closed-canopy forest and the other one living in a more open $C_{3}$ environment.

The potential of ${ }^{13} \mathrm{C}$ measurement of herbivore tissue for tracking the degree of closure of habitat is an important question to address. Indeed, climatic fluctuations in the past has led to major vegetation changes, as it was the case of the transition from Late-Glacial to Early Holocene (ca 15,000-6000 years cal BP) in western Europe. As a result of global climatic warming, the vegetation composition changed drastically from steppe-tundra dominated by grass and herbaceous dicotyledones to temperate dense deciduous forest through intermediate stages of open boreal-like forests as reconstructed from pollen records (e.g. Amman and Lotter, 1989; Beaulieu et al., 1994a,b). Among game species, arctic-steppe species like reindeer (Rangifer tarandus) and bison (Bison priscus) were gradually replaced by temperate species like aurochs (Bos primigenius), red deer (Cervus elaphus) and roe deer (Capreolus capreolus) after a period of coexistence during the Late Glacial Interstadial. Late-glacial and Early Holocene major change of landscape provoked a change in the subsistence strategies of prehistoric populations linked to the change in the habitat of their game. Therefore, it is of high interest to define the degree of opening of the habitat of ancient herbivores using their $\delta^{13} \mathrm{C}$ values. In this context, the decrease in herbivore ${ }^{13} \mathrm{C}$ abundances during Late-Glacial-Early Holocene transition in western Europe has been attributed to the increasing forest cover at this time (Drucker et al., 2003; Noe-Nyggard et al., 2005). In contrast, some papers consider that the decrease in ${ }^{13} \mathrm{C}$ abundance of herbivores during this period essentially results from an increase in $\mathrm{CO}_{2}$ concentration in the atmosphere along with a decrease in plant ${ }^{13} \mathrm{C}$ abundance (Richards and Hedges, 2003; Stevens and Hedges, 2004).
We wanted to determine whether the canopy effect is a convincing explanation for the observed decrease in carbon-13 abundance in herbivores during the Late-Glacial/Early Holocene transition. For this purpose, we address the following two questions. First, in modern boreal and temperate environments, can the canopy effect result in depletion of ${ }^{13} \mathrm{C}$ abundance in tissues of large herbivores regardless of their forage preferences? Second, during the Late-Glacial and Early Holocene, did the pattern and timing of the decline in ${ }^{13} \mathrm{C}$ abundance in herbivores vary according to species and geographical location? Such variation is expected if a local parameter, such as canopy effect, rather than a global parameter, such as atmospheric $\mathrm{CO}_{2}$ content, is primarily involved in the herbivore ${ }^{13} \mathrm{C}$ depletion.

\section{Background information on habitat and dietary specialisation of the studied ruminants}

The present study includes carbon isotopic data from modern representatives of five herbivore species, all belonging to the Artiodactyla and more specifically to the Ruminantia. Three of them are cervids: reindeer (Rangifer tarandus), roe deer (Capreolus capreolus), red deer (Cervus elaphus). The two others are bovini: American bison (Bison bison), and European wisent (Bison bonasus). These species have been selected on the basis of several factors: (1) a similar digestive physiology based on rumination, (2) well-monitored populations with minimal anthropogenic interference, living under closed-canopy and more open conditions (reindeer, roe deer, red deer), (3) remains of such taxa are often abundant in the Late-Glacial and/or Early Holocene archaeological sites from France. Two extinct bovini are also considered here, steppe bison (Bison priscus) and aurochs (Bos primigenius), and ancient domestic cattle (Bos taurus), which was used for comparison with its wild relatives during the Early Holocene.

Reindeer (Rangifer tarandus) is a medium-sized deer adapted to arctic and boreal environments, with a circum-polar modern distribution. In Canada, two main ecotypes are recognized, one dwelling essentially in the arctic tundra, the barren-ground caribou, the other one dwelling all year long in old-growth mature boreal forest, the woodland caribou (e.g. Kelsall, 1968; Russell, 1998). Wild reindeer from Siberia dwell in varying habitats, from arctic tundra to more forested environments. Reindeer are mixed feeders, quite plastic in their food habits. They consume a large range of vascular plants, including shrub leaves, forbs, and graminoids (grass, carex). In contrast with other herbivores, reindeer have the ability to sustain primarily on large amounts of lichen, in tundra as well as in boreal forest (e.g., Flerov, 1952; Kelsall, 1968; Lindgren et al., 1983; Rettie et al., 1997; Russell, 1998; de Bellefeuille, 2001). Living outside the geographical range of cultivated areas, caribou from mainland North America is one of the few deer with populations dwelling in contrasted habitats with similar diet composition, but without significant anthropogenic interferences. It is therefore a good candidate to test the impact of canopy effect on $\delta^{13} \mathrm{C}$ values. Nonetheless this species disappears from the fossil record at the end of the Late-Glacial, around 12,000 years ago in France (e.g., Bridault et al., 2000), whereas it is still documented during the last cold oscillation of the Late-Glacial in Belgium and northern Germany (Charles, 1993; Baales,1996) and at the beginning of Holocene in Great Britain and Southern Scandinavia (e.g., Coard and Chamberlain, 1999; Ukkonen et al., 2006; Aaris-Sorensen et al., 2007).

Red deer (Cervus elaphus), called elk in Eurasia and wapiti in North America, is a large sized deer with great ecological plasticity, living under a large diversity of habitats including steppe, boreal and closed temperate forests (Geist, 1999). It is an opportunistic mixed feeder (Hoffmann, 1989), whose diet includes edible portions of trees and shrubs, forbs, grass and sedges (e.g., Gebczynska, 1980; Heptner et al., 1989; Gebert and Verheyden-Tixier, 2001). European red deer tend to favour forbs, foliage and browse, while Siberian and American red deer consume higher proportions of grass (Geist, 1999). In Europe, 


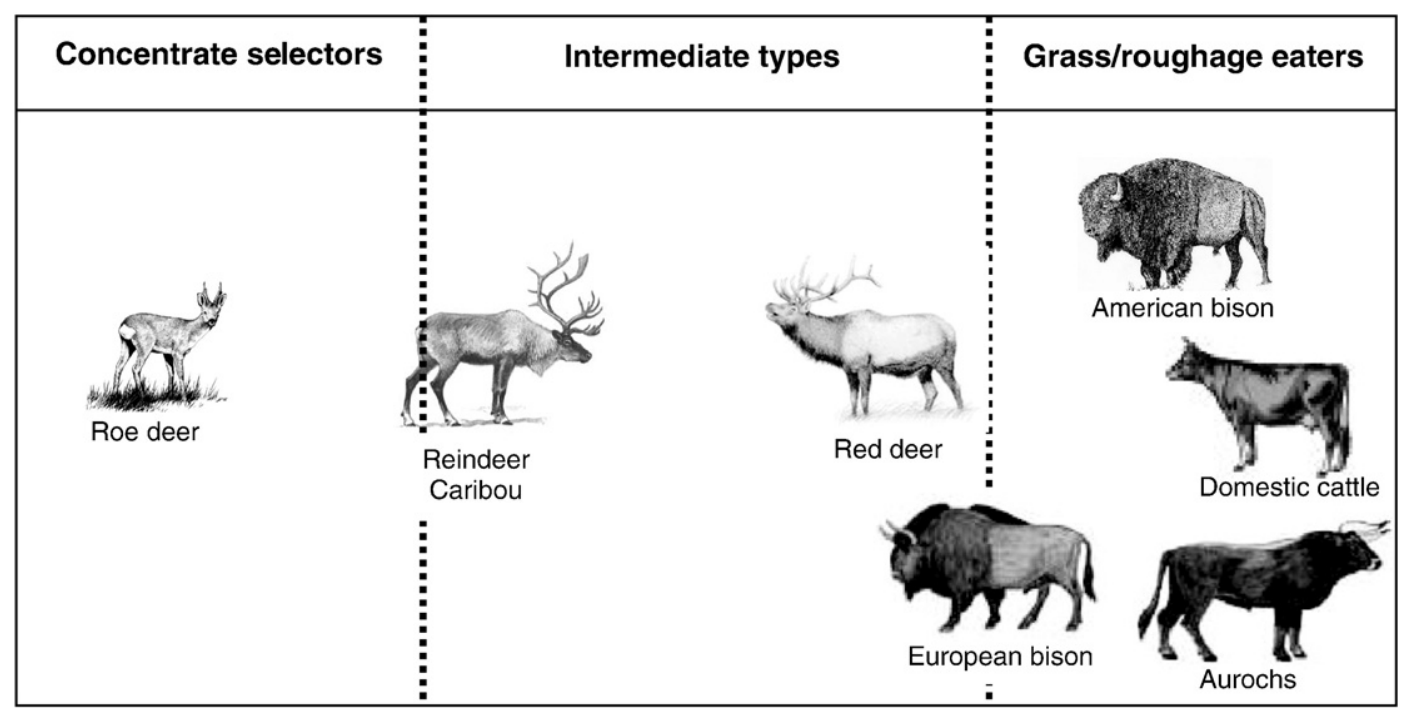

Fig. 1. Dietary classification of the studied species (modified from Hoffmann, 1989; Kie et al., 2003).

most red deer populations live close to areas of human activities, making it very difficult to avoid anthropogenic interference. However, this species was present in many parts of France during the Late Pleistocene and the Holocene without discontinuity.

Roe deer (Capreolus capreolus) is a small deer ranging from Europe to Siberia (Geist, 1999). They are specialised concentrate feeders and consume selectively the nutrient-rich parts of plants (Hoffmann, 1989). They may live in a wide variety of habitats, occurring in almost all of the natural habitats found in Europe, including deciduous, coniferous and Mediterranean forests, shrublands, moorlands and marshes. Only high alpine areas and the most open grasslands are rarely occupied (Linnell et al., 1998; Geist, 1999). As for red deer, most European roe deer populations live close to areas of human activities, making it very difficult to avoid anthropogenic interference. This species was present in southern France since the beginning of the Bölling (e.g., Hufthammer and Aaris-Sorensen, 1998) and expanded later in northern areas (Bridault and Chaix, 2002).

The bovini considered here belong to several species: American bison (Bison bison), European wisent (Bison bonasus), steppe bison (Bison priscus) and aurochs (Bos primigenius). The last two species, nowadays extinct, are present in the Late-glacial and early Holocene deposits of France. Both modern bison species have narrowly escaped extinction during historical periods and correspond to relic or reintroduced populations. The American bison is found in grassland and woodland habitats in USA and Canada. American bison are grazers that consume essentially graminoids (i.e. grass and sedges) (e.g.,
Plumb and Dodd, 1993; Coppedege et al., 1998; Fortin et al., 2003). European bison includes some browse in their diet, but a majority of their forage is composed of graminoids (Pucek et al., 2002). Among ruminants, it is classified as a grass/roughage eater (Hoffmann, 1989). The extinct steppe bison Bison priscus and the aurochs Bos primigenius were both grass eaters with slightly different ecological preferences, drier steppes in the case of steppe bison and more wooded and humid contexts for aurochs (von Koenigswald, 1999).

An additional advantage to choose these species is the possibility to compare carbon isotopic results for specialised feeders, such as roe deer, with those of more generalists, such as red deer and bison, in the same environment. The dietary characteristics of these species are summarized in Fig. 1. It will be therefore possible to compare the carbon isotope variations for species which do not change their diet between open and closed habitats and for species which can change their diet when they change habitat.

\section{Materials and methods}

To investigate evidence for the canopy effect in high and mid-latitude environments, we used carbon isotopic data from herbivores of modern ecosystems ranging from open tundra to dense temperate forest. We concentrated our efforts on those ecosystems for which a maximum of information was available about the ecology of the animals, especially their diet and the plant formations where they live, and about the carbon isotopic data of animals and the plants they fed on.

Table 1

Description of the modern ecosystems selected to investigate the impact of the canopy effect on carbon-13 abundances in plants and herbivores

\begin{tabular}{|c|c|c|c|c|}
\hline Taxon & Material & Location-temperature-precipitation & Environment & References \\
\hline Reindeer & Collagen & $\begin{array}{l}\text { Qamanirjuaq (Nunavut, Canada) } 0-200 \mathrm{~m} \text { asl, } 60-64^{\circ} \mathrm{N} \text {, } \\
93-97^{\circ} \mathrm{W} \text { Min temp }=-32{ }^{\circ} \mathrm{C} \text {, } \max \text { temp }=+5{ }^{\circ} \mathrm{C} \text { Annual prec. }<250 \mathrm{~mm} / \text { year }\end{array}$ & Arctic tundra & $\begin{array}{l}\text { Drucker et al. } \\
\text { (2001) }\end{array}$ \\
\hline Reindeer & Hair & $\begin{array}{l}\text { Queen Maud Gulf (Nunavut, Canada) } 200-500 \mathrm{~m} \text { asl, } 60-68^{\circ} \mathrm{N}, 105-120^{\circ} \mathrm{W} \text { Min } \\
\text { temp }=-32{ }^{\circ} \mathrm{C} \text {, max temp }=+5{ }^{\circ} \mathrm{C} \text { Annual prec. }<250 \mathrm{~mm} / \text { year }\end{array}$ & Arctic tundra & This study \\
\hline $\begin{array}{l}\text { Red deer American } \\
\text { bison Reindeer }\end{array}$ & Hair & $\begin{array}{l}\text { Central Saskatchewan, Canada } 53^{\circ} 63^{\prime} \mathrm{N}, 106^{\circ} 2 \text { 'W Mean temp }=0.4{ }^{\circ} \mathrm{C} \text {, } \\
\text { min temp }=-25{ }^{\circ} \mathrm{C} \text {, max temp }=+25^{\circ} \mathrm{C} \text { Annual prec. }=400-500 \mathrm{~mm} / \text { year }\end{array}$ & $\begin{array}{l}\text { boreal forest, primarily aspen, } \\
\text { spruce, pine, poplar }\end{array}$ & $\begin{array}{l}\text { Urton and } \\
\text { Hobson, } 2005\end{array}$ \\
\hline Reindeer & Collagen & $\begin{array}{l}\text { Northeastern Yakutia, } 0-200 \mathrm{~m}, 66^{\circ} \mathrm{N}, 150^{\circ} \mathrm{E} \text { Min temp }=-40^{\circ} \mathrm{C} \\
\text { max temp }=+13^{\circ} \mathrm{C} \text { Annual prec. }=200 \mathrm{~mm} / \text { year }\end{array}$ & Open taiga/tundra & $\begin{array}{l}\text { Bocherens et } \\
\text { al., } 1996\end{array}$ \\
\hline Red deer Roe deer & Collagen & $\begin{array}{l}\text { Cis-Baikal, Siberia, } 400-3000 \mathrm{~m} \text { asl, } 51^{\circ}-56^{\circ} \mathrm{N}, 102^{\circ}-110^{\circ} \mathrm{E} \text { Mean temp }=-0.7^{\circ} \mathrm{C} \text {, } \\
\text { Min temp }=-30{ }^{\circ} \mathrm{C} \text {, max temp }=+25^{\circ} \mathrm{C} \text { Annual prec. }=495 \mathrm{~mm} / \text { year }\end{array}$ & $\begin{array}{l}\text { boreal forest/steppe transitional zone } \\
\text { primarily pines, larches, cedars, spruces, } \\
\text { firs with a mixture of poplar, birch, aspen }\end{array}$ & $\begin{array}{l}\text { Weber et al., } \\
2002\end{array}$ \\
\hline $\begin{array}{l}\text { Red deer Roe deer } \\
\text { European bison }\end{array}$ & Collagen & $\begin{array}{l}\text { Bialowieza Forest, Poland, } 147-172 \mathrm{~m} \text { asl } 52^{\circ} 43^{\prime} \mathrm{N}, 23^{\circ} 50 \text { 'E mean } \\
\text { temp }=6.8^{\circ} \mathrm{C} \text {, min temp }=-4.7^{\circ} \mathrm{C} \text {, } \max \text { temp }=+17.8^{\circ} \mathrm{C} \\
\text { annual prec. }=641 \mathrm{~mm} / \text { year }\end{array}$ & $\begin{array}{l}\text { temperate deciduous forest zone } \\
\text { primarily oak, lime, hornbeam with a } \\
\text { mixture of ash, maple and elm }\end{array}$ & $\begin{array}{l}\text { Bocherens and } \\
\text { Drucker, } 2003 \text {, } \\
\text { and this work }\end{array}$ \\
\hline Roe deer & Collagen & $\begin{array}{l}\text { Dourdan Forest, France, } 100 \mathrm{~m} \text { asl } 48^{\circ} 19^{\prime} \mathrm{N}, 2^{\circ} 01^{\prime} \mathrm{E} \text { mean temp }=11.7^{\circ} \mathrm{C} \text {, } \\
\text { min temp }=3{ }^{\circ} \mathrm{C} \text {, max temp }=18.5^{\circ} \mathrm{C} \text { annual prec. }=630 \mathrm{~mm} / \text { year }\end{array}$ & $\begin{array}{l}\text { temperate deciduous forest, primarily } \\
\text { beech }\end{array}$ & $\begin{array}{l}\text { Rodière et al., } \\
1996\end{array}$ \\
\hline
\end{tabular}




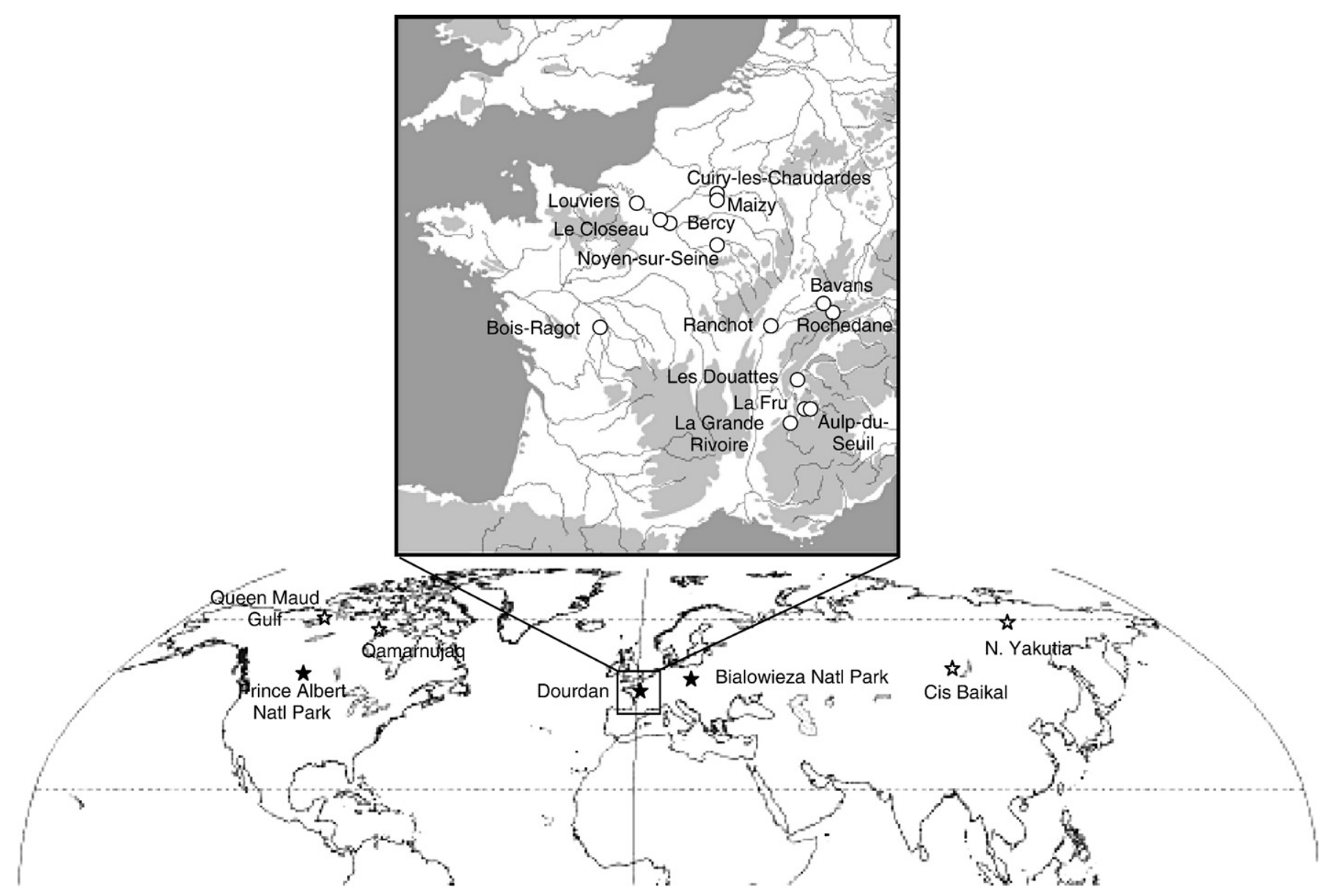

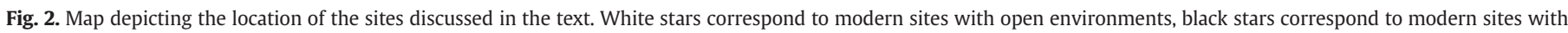
closed-canopy environments, white circles correspond to archaeological sites with faunal material documenting the late Glacial to Holocene transition in France.

The selected modern specimens and their origin are described in detail in Table 1 and the location of the sites is shown on Fig. 2. To avoid anthropogenic influences on the isotopic record of the diet as much as possible, our selected large herbivores were free-ranging wild animals. Bone and hair material of modern ruminants comes from seven localities in North America and Eurasia (Table 1; Fig. 2). Caribou and reindeer from open (Queen Maud Gulf, Qamanirjuaq, Northern Yakutia) and closed forested (Central Saskatchewan) environments have been selected. Red deer specimens are from closed forested environments (Central Saskatchewan and Bialowieza Forest National Park) and more open environments (Cis-Baikal). Roe deer specimens are also from closed forested environments (Dourdan forest and Bialowieza Forest National Park) and more open environments (Cis-Baikal). Bison specimens are from closed forest environments (Central Saskatchewan and Bialowieza Forest National Park). Except for Queen Maud Gulf caribou which are unpublished data, all of the isotopic data were previously published in various works (Table 1). In the present paper, they were corrected from variations of $\delta^{13} \mathrm{C}$ values in atmospheric $\mathrm{CO}_{2}$ between 1966 and 2003.

Isotopic data from modern large herbivores were obtained for hair keratin and bone collagen, involving different diet-tissue isotopic discrimination factors, which prevented direct comparison of $\delta^{13} \mathrm{C}$ values. Therefore, measured $\delta^{13} \mathrm{C}$ values were converted to diet $\delta^{13} \mathrm{C}$ values $\left(\delta^{13} \mathrm{C}_{\text {diet }}\right)$ using the average fractionation factor between the mean diet and the considered tissue inferred from the literature (Vogel, 1978; van der Merwe, 1989; Ambrose, 1993; Rodière et al., 1996; Sponheimer et al., 2003; Ayliffe et al., 2004) and summarised in Table 2. Moreover, since isotopic analyses were performed on specimens living at different times, we took into account the rapid changes in $\delta^{13} \mathrm{C}$ value of atmospheric $\mathrm{CO}_{2}$ linked to modern industrial activities and deforestation. The $\delta^{13} \mathrm{C}_{\text {diet }}$ values were thus set to a similar atmospheric $\delta^{13} \mathrm{C}$ value of $-7 \%$, the average value during Upper Pleistocene times (Leuenberger et al., 1992) by using a correction formula presented by Feng (1998).

To examine the decline in $\delta^{13} \mathrm{C}$ values of different species during the Late-Glacial/Early Holocene transition, we selected ancient bone specimens of cervids, red deer and roe deer, and large bovines, namely wild bovine and domestic cattle, from a restricted geographic area, the Paris Basin. The material comes from seven sites, with chronological

Table 2

Review of ${ }^{13} \mathrm{C}$ discrimination between hair and collagen of modern large herbivores and their diet

\begin{tabular}{llll}
\hline Tissue & Taxon & $\Delta \delta^{13} \mathrm{C}$ tissue-diet & Reference \\
\hline Hair & Horse & 2.7 & Ayliffe et al., 2004 \\
Hair & Cattle & 2.7 & Sponheimer et al., 2003 \\
Hair & Goat & 3.2 & Sponheimer et al., 2003 \\
Hair & Alpaca & 3.2 & Sponheimer et al., 2003 \\
Hair & Llama & 3.5 & Sponheimer et al., 2003 \\
Hair & Rabbit & 3.4 & Sponheimer et al., 2003 \\
& MEAN & 3.1 & \\
& SD & 0.3 & \\
Collagen & Bison & 5.0 & Ambrose, 1993 \\
Collagen & Giraffe & 5.5 & Ambrose, 1993 \\
Collagen & $C_{3}$ eater herbivores & 5.0 & van der Merwe, 1989 \\
Collagen & $C_{3}$ eater herbivores & 5.3 & Vogel, 1978a \\
Collagen & Roe deer & 4.5 & Rodière et al., 1996 \\
& MEAN & 5.1 & \\
& SD & 0.3 & \\
\hline
\end{tabular}


Table 3

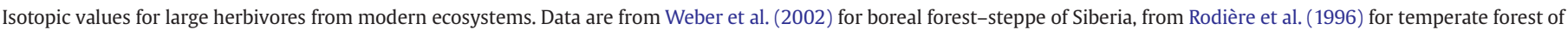
Dourdan, and from Urton and Hobson (2005) for boreal forest of western Canada

\begin{tabular}{|c|c|c|c|c|c|c|c|c|c|c|}
\hline Species & $\operatorname{Lab~n}^{\circ}$ & Site & Habitat & Tissue & Year & $\begin{array}{l}\delta^{13} \mathrm{C} \\
\text { tissue }\end{array}$ & $\begin{array}{l}\delta^{13} \mathrm{C} \text { diet calculated } \\
\delta^{13} \mathrm{C} \text { coll }-5.1 \\
\delta^{13} \mathrm{C} \text { hair }-3.1\end{array}$ & $\Delta{ }^{13} \mathrm{C}$ atm & $\begin{array}{l}\delta^{13} \mathrm{C} \text { diet } \\
\text { Calculated } \\
\text { corrected }\end{array}$ & Ref. \\
\hline Reindeer & 204 & Qamanirjuaq, Nunavut & Arctic tundra & Collagen & 1966 & -19.4 & -24.5 & 0.24 & -24.2 & Drucker et al. (2001) \\
\hline Reindeer & 216 & Qamanirjuaq, Nunavut & Arctic tundra & Collagen & 1966 & -19.5 & -24.6 & 0.24 & -24.3 & Drucker et al. (2001) \\
\hline Reindeer & 692 & Qamanirjuaq, Nunavut & Arctic tundra & Collagen & 1967 & -19.2 & -24.3 & 0.26 & -24.0 & Drucker et al. (2001) \\
\hline Reindeer & 80 & Qamanirjuaq, Nunavut & Arctic tundra & Collagen & 1966 & -20.0 & -25.1 & 0.24 & -24.8 & Drucker et al. (2001) \\
\hline Reindeer & $397-1$ & Qamanirjuaq, Nunavut & Arctic tundra & Collagen & 1967 & -19.6 & -24.7 & 0.26 & -24.4 & Drucker et al. (2001) \\
\hline Reindeer & $397-2$ & Qamanirjuaq, Nunavut & Arctic tundra & Collagen & 1967 & -18.8 & -23.9 & 0.26 & -23.6 & Drucker et al. (2001) \\
\hline Reindeer & 22882 & Queen Maud Gulf, Nunavut & Arctic tundra & Hair & 2002 & -22.4 & -25.5 & 1.20 & -24.3 & This work \\
\hline Reindeer & 22883 & Queen Maud Gulf, Nunavut & Arctic tundra & Hair & 2002 & -23.0 & -26.1 & 1.20 & -24.9 & This work \\
\hline Reindeer & 22884 & Queen Maud Gulf, Nunavut & Arctic tundra & Hair & 2002 & -22.0 & -25.1 & 1.20 & -23.9 & This work \\
\hline Reindeer & 22885 & Queen Maud Gulf, Nunavut & Arctic tundra & Hair & 2002 & -22.4 & -25.5 & 1.20 & -24.3 & This work \\
\hline Reindeer & 22886 & Queen Maud Gulf, Nunavut & Arctic tundra & Hair & 2002 & -22.6 & -25.7 & 1.20 & -24.5 & This work \\
\hline Reindeer & 22968 & Queen Maud Gulf, Nunavut & Arctic tundra & Hair & 2003 & -22.9 & -26.0 & 1.23 & -24.7 & This work \\
\hline Reindeer & 22969 & Queen Maud Gulf, Nunavut & Arctic tundra & Hair & 2003 & -23.1 & -26.2 & 1.23 & -24.9 & This work \\
\hline Reindeer & 22970 & Queen Maud Gulf, Nunavut & Arctic tundra & Hair & 2003 & -22.5 & -25.6 & 1.23 & -24.3 & This work \\
\hline Reindeer & 22971 & Queen Maud Gulf, Nunavut & Arctic tundra & Hair & 2003 & -22.4 & -25.5 & 1.23 & -24.3 & This work \\
\hline Reindeer & 22972 & Queen Maud Gulf, Nunavut & Arctic tundra & Hair & 2003 & -22.8 & -25.9 & 1.23 & -24.7 & This work \\
\hline Reindeer & 22973 & Queen Maud Gulf, Nunavut & Arctic tundra & Hair & 2003 & -22.8 & -25.9 & 1.23 & -24.7 & This work \\
\hline Reindeer & 22974 & Queen Maud Gulf, Nunavut & Arctic tundra & Hair & 2003 & -23.1 & -26.2 & 1.23 & -24.9 & This work \\
\hline Reindeer & 22975 & Queen Maud Gulf, Nunavut & Arctic tundra & Hair & 2003 & -22.0 & -25.1 & 1.23 & -23.9 & This work \\
\hline Reindeer & 22976 & Queen Maud Gulf, Nunavut & Arctic tundra & Hair & 2003 & -22.9 & -26.0 & 1.23 & -24.8 & This work \\
\hline Reindeer & 22977 & Queen Maud Gulf, Nunavut & Arctic tundra & Hair & 2003 & -22.4 & -25.5 & 1.23 & -24.2 & This work \\
\hline Reindeer & 22978 & Queen Maud Gulf, Nunavut & Arctic tundra & Hair & 2003 & -21.9 & -25.0 & 1.23 & -23.8 & This work \\
\hline Reindeer & 22980 & Queen Maud Gulf, Nunavut & Arctic tundra & Hair & 2003 & -23.4 & -26.5 & 1.23 & -25.3 & This work \\
\hline Reindeer & 22981 & Queen Maud Gulf, Nunavut & Arctic tundra & Hair & 2003 & -23.0 & -26.1 & 1.23 & -24.9 & This work \\
\hline Reindeer & 22982 & Queen Maud Gulf, Nunavut & Arctic tundra & Hair & 2003 & -22.8 & -25.9 & 1.23 & -24.6 & This work \\
\hline Reindeer & 22983 & Queen Maud Gulf, Nunavut & Arctic tundra & Hair & 2003 & -22.3 & -25.4 & 1.23 & -24.1 & This work \\
\hline Reindeer & 22984 & Queen Maud Gulf, Nunavut & Arctic tundra & Hair & 2003 & -23.0 & -26.1 & 1.23 & -24.9 & This work \\
\hline Reindeer & 22985 & Queen Maud Gulf, Nunavut & Arctic tundra & Hair & 2003 & -22.2 & -25.3 & 1.23 & -24.0 & This work \\
\hline Reindeer & 28053 & Queen Maud Gulf, Nunavut & Arctic tundra & Hair & 2003 & -22.1 & -25.2 & 1.23 & -23.9 & This work \\
\hline Reindeer & 28054 & Queen Maud Gulf, Nunavut & Arctic tundra & Hair & 2003 & -21.7 & -24.8 & 1.23 & -23.6 & This work \\
\hline Reindeer & 22924 & Central Saskatchewan & Boreal forest & Hair & 1992 & -23.4 & -26.5 & 0.85 & -25.6 & Urton and Hobson (2005) \\
\hline Reindeer & 22925 & Central Saskatchewan & Boreal forest & Hair & 1992 & -23.7 & -26.8 & 0.85 & -25.9 & Urton and Hobson (2005) \\
\hline Reindeer & 22928 & Central Saskatchewan & Boreal forest & Hair & 1993 & -23.7 & -26.8 & 0.88 & -25.9 & Urton and Hobson (2005) \\
\hline Reindeer & 22931 & Central Saskatchewan & Boreal forest & Hair & 1996 & -23.6 & -26.7 & 0.98 & -25.7 & Urton and Hobson (2005) \\
\hline Reindeer & 22936 & Central Saskatchewan & Boreal forest & Hair & 1994 & -23.8 & -26.9 & 0.91 & -25.9 & Urton and Hobson (2005) \\
\hline Reindeer & 22939 & Central Saskatchewan & Boreal forest & Hair & 1994 & -23.6 & -26.7 & 0.91 & -25.8 & Urton and Hobson (2005) \\
\hline Reindeer & 22940 & Central Saskatchewan & Boreal forest & Hair & 1994 & -23.3 & -26.4 & 0.91 & -25.5 & Urton and Hobson (2005) \\
\hline Reindeer & 22943 & Central Saskatchewan & Boreal forest & Hair & 1994 & -23.3 & -26.4 & 0.91 & -25.5 & Urton and Hobson (2005) \\
\hline Reindeer & 22946 & Central Saskatchewan & Boreal forest & Hair & 1994 & -22.9 & -26.0 & 0.91 & -25.1 & Urton and Hobson (2005) \\
\hline Reindeer & 22948 & Central Saskatchewan & Boreal forest & Hair & 1994 & -23.8 & -26.9 & 0.91 & -26.0 & Urton and Hobson (2005) \\
\hline Reindeer & 22949 & Central Saskatchewan & Boreal forest & Hair & 1995 & -23.5 & -26.6 & 0.95 & -25.6 & Urton and Hobson (2005) \\
\hline Reindeer & 22951 & Central Saskatchewan & Boreal forest & Hair & 1995 & -24.0 & -27.1 & 0.95 & -26.1 & Urton and Hobson (2005) \\
\hline Reindeer & 22957 & Central Saskatchewan & Boreal forest & Hair & 1995 & -23.3 & -26.4 & 0.95 & -25.4 & Urton and Hobson (2005) \\
\hline Reindeer & 22958 & Central Saskatchewan & Boreal forest & Hair & 1995 & -22.6 & -25.7 & 0.95 & -24.7 & Urton and Hobson (2005) \\
\hline Reindeer & 101300 & Northeastern Yakutia & Open taiga & Collagen & 1994 & -19.6 & -24.7 & 0.91 & -23.8 & Bocherens et al. (1996) \\
\hline Reindeer & 102300 & Northeastern Yakutia & Open taiga & Collagen & 1994 & -20.2 & -25.3 & 0.91 & -24.4 & Bocherens et al. (1996) \\
\hline Reindeer & 102800 & Northeastern Yakutia & Open taiga & Collagen & 1994 & -19.4 & -24.5 & 0.91 & -23.6 & Bocherens et al. (1996) \\
\hline Reindeer & 102900 & Northeastern Yakutia & Open taiga & Collagen & 1994 & -20.2 & -25.3 & 0.91 & -24.4 & Bocherens et al. (1996) \\
\hline Reindeer & 103000 & Northeastern Yakutia & Open taiga & Collagen & 1994 & -19.5 & -24.6 & 0.91 & -23.7 & Bocherens et al. (1996) \\
\hline Red deer & 17258 & Central Saskatchewan & Boreal forest & Hair & 2002 & -25.0 & -28.1 & 1.20 & -26.9 & Urton and Hobson (2005) \\
\hline Red deer & 17259 & Central Saskatchewan & Boreal forest & Hair & 2002 & -24.8 & -27.9 & 1.20 & -26.7 & Urton and Hobson (2005) \\
\hline Red deer & 17903 & Central Saskatchewan & Boreal forest & Hair & 2002 & -26.5 & -29.6 & 1.20 & -28.4 & Urton and Hobson (2005) \\
\hline Red deer & 17904 & Central Saskatchewan & Boreal forest & Hair & 2002 & -26.3 & -29.4 & 1.20 & -28.2 & Urton and Hobson (2005) \\
\hline Red deer & 17906 & Central Saskatchewan & Boreal forest & Hair & 2002 & -25.6 & -28.7 & 1.20 & -27.5 & Urton and Hobson (2005) \\
\hline Red deer & 17907 & Central Saskatchewan & Boreal forest & Hair & 2002 & -26.6 & -29.7 & 1.20 & -28.5 & Urton and Hobson (2005) \\
\hline Red deer & 19986 & Central Saskatchewan & Boreal forest & Hair & 2002 & -27.1 & -30.2 & 1.20 & -29.0 & Urton and Hobson (2005) \\
\hline Red deer & 22557 & Central Saskatchewan & Boreal forest & Hair & 2002 & -24.2 & -27.3 & 1.20 & -26.1 & Urton and Hobson (2005) \\
\hline Red deer & 22558 & Central Saskatchewan & Boreal forest & Hair & 2002 & -24.3 & -27.4 & 1.20 & -26.2 & Urton and Hobson (2005) \\
\hline Red deer & 93.052 & Cis-Baikal & Forest-steppe & Collagen & 2000 & -22.4 & -27.5 & 1.12 & -26.4 & Weber et al. (2002) \\
\hline Red deer & 93.053 & Cis-Baikal & Forest-steppe & Collagen & 2000 & -20.4 & -25.5 & 1.12 & -24.4 & Weber et al. (2002) \\
\hline Red deer & 93.066 & Cis-Baikal & Forest-steppe & Collagen & 2000 & -22.7 & -27.8 & 1.12 & -26.7 & Weber et al. (2002) \\
\hline Red deer & 93.149 & Cis-Baikal & Forest-steppe & Collagen & 2000 & -22.1 & -27.2 & 1.12 & -26.1 & Weber et al. (2002) \\
\hline Red deer & BLW700 & Bialowieza Forest & Deciduous forest & Collagen & 1970 & -21.7 & -26.8 & 0.31 & -26.5 & This work \\
\hline Red deer & BLW800 & Bialowieza Forest & Deciduous forest & Collagen & 1970 & -24.2 & -29.3 & 0.31 & -29.0 & This work \\
\hline Red deer & BLW900 & Bialowieza Forest & Deciduous forest & Collagen & 1970 & -24.1 & -29.2 & 0.31 & -28.9 & This work \\
\hline Red deer & BLW1000 & Bialowieza Forest & Deciduous forest & Collagen & 1970 & -23.9 & -29.0 & 0.31 & -28.7 & This work \\
\hline Red deer & BLW1100 & Bialowieza Forest & Deciduous forest & Collagen & 1970 & -23.1 & -28.2 & 0.31 & -27.9 & This work \\
\hline Red deer & ACT500 & Bialowieza Forest & Deciduous forest & Collagen & 1994 & -24.2 & -29.3 & 0.91 & -28.4 & This work \\
\hline Roe deer & 93.057 & Cis-Baikal & Forest-steppe & Collagen & 2000 & -20.6 & -25.7 & 1.12 & -24.6 & Weber et al. (2002) \\
\hline Roe deer & 93.058 & Cis-Baikal & Forest-steppe & Collagen & 2000 & -20 & -25.1 & 1.12 & -24.0 & Weber et al. (2002) \\
\hline Roe deer & 93.059 & Cis-Baikal & Forest-steppe & Collagen & 2000 & -21.6 & -26.7 & 1.12 & -25.6 & Weber et al. (2002) \\
\hline
\end{tabular}


Table 3 (continued)

\begin{tabular}{|c|c|c|c|c|c|c|c|c|c|c|}
\hline Species & $\operatorname{Lab}^{\circ}$ & Site & Habitat & Tissue & Year & $\begin{array}{l}\delta^{13} \mathrm{C} \\
\text { tissue }\end{array}$ & $\begin{array}{l}\delta^{13} \mathrm{C} \text { diet calculated } \\
\delta^{13} \mathrm{C} \text { coll }-5.1 \\
\delta^{13} \mathrm{C} \text { hair }-3.1\end{array}$ & $\Delta^{13} \mathrm{C}$ atm & $\begin{array}{l}\delta^{13} \mathrm{C} \text { diet } \\
\text { Calculated } \\
\text { corrected }\end{array}$ & Ref. \\
\hline Roe deer & 93.060 & Cis-Baikal & Forest-steppe & Collagen & 2000 & -20.4 & -25.5 & 1.12 & -24.4 & Weber et al. (2002) \\
\hline Roe deer & 93.061 & Cis-Baikal & Forest-steppe & Collagen & 2000 & -20.4 & -25.5 & 1.12 & -24.4 & Weber et al. (2002) \\
\hline Roe deer & 93.062 & Cis-Baikal & Forest-steppe & Collagen & 2000 & -22.9 & -28.0 & 1.12 & -26.9 & Weber et al. (2002) \\
\hline Roe deer & 1204 & Dourdan Forest & Deciduous forest & Collagen & 1990 & -23.4 & -28.5 & 0.79 & -27.7 & Rodière et al. (1996) \\
\hline Roe deer & 7200 & Dourdan Forest & Deciduous forest & Collagen & 1990 & -25.6 & -30.7 & 0.79 & -29.9 & Rodière et al. (1996) \\
\hline Roe deer & 9200 & Dourdan Forest & Deciduous forest & Collagen & 1990 & -24.7 & -29.8 & 0.79 & -29.0 & Rodière et al. (1996) \\
\hline Roe deer & 10200 & Dourdan Forest & Deciduous forest & Collagen & 1990 & -25.1 & -30.2 & 0.79 & -29.4 & Rodière et al. (1996) \\
\hline Roe deer & 11200 & Dourdan Forest & Deciduous forest & Collagen & 1990 & -26.0 & -31.1 & 0.79 & -30.3 & Rodière et al. (1996) \\
\hline Roe deer & 14200 & Dourdan Forest & Deciduous forest & Collagen & 1990 & -23.8 & -28.9 & 0.79 & -28.1 & Rodière et al. (1996) \\
\hline Roe deer & 15200 & Dourdan Forest & Deciduous forest & Collagen & 1990 & -25.7 & -30.8 & 0.79 & -30.0 & Rodière et al. (1996) \\
\hline Roe deer & 20200 & Dourdan Forest & Deciduous forest & Collagen & 1990 & -23.8 & -28.9 & 0.79 & -28.1 & Rodière et al. (1996) \\
\hline Roe deer & 22200 & Dourdan Forest & Deciduous forest & Collagen & 1990 & -26.1 & -31.2 & 0.79 & -30.4 & Rodière et al. (1996) \\
\hline Roe deer & 23200 & Dourdan Forest & Deciduous forest & Collagen & 1990 & -24.9 & -30.0 & 0.79 & -29.2 & Rodière et al. (1996) \\
\hline Roe deer & 24200 & Dourdan Forest & Deciduous forest & Collagen & 1990 & -25.7 & -30.8 & 0.79 & -30.0 & Rodière et al. (1996) \\
\hline Roe deer & 25200 & Dourdan Forest & Deciduous forest & Collagen & 1990 & -24.9 & -30.0 & 0.79 & -29.2 & Rodière et al. (1996) \\
\hline Roe deer & 26200 & Dourdan Forest & Deciduous forest & Collagen & 1990 & -25.6 & -30.7 & 0.79 & -29.9 & Rodière et al. (1996) \\
\hline Roe deer & 27200 & Dourdan Forest & Deciduous forest & Collagen & 1990 & -23.5 & -28.6 & 0.79 & -27.8 & Rodière et al. (1996) \\
\hline Roe deer & 28200 & Dourdan Forest & Deciduous forest & Collagen & 1990 & -24.1 & -29.2 & 0.79 & -28.4 & Rodière et al. (1996) \\
\hline Roe deer & 29200 & Dourdan Forest & Deciduous forest & Collagen & 1990 & -23.8 & -28.9 & 0.79 & -28.1 & Rodière et al. (1996) \\
\hline Roe deer & 30200 & Dourdan Forest & Deciduous forest & Collagen & 1990 & -24.9 & -30.0 & 0.79 & -29.2 & Rodière et al. (1996) \\
\hline Roe deer & 2203 & Dourdan Forest & Deciduous forest & Collagen & 1990 & -23.7 & -28.8 & 0.79 & -28.0 & Rodière et al. (1996) \\
\hline Roe deer & BLW1200 & Bialowieza Forest & Deciduous forest & Collagen & 1969 & -23.2 & -28.3 & 0.29 & -28.0 & Bocherens and Drucker (2003) \\
\hline Roe deer & BLW1300 & Bialowieza Forest & Deciduous forest & Collagen & 1970 & -24.5 & -29.6 & 0.31 & -29.3 & Bocherens and Drucker (2003) \\
\hline Roe deer & BLW1400 & Bialowieza Forest & Deciduous forest & Collagen & 1994 & -23.5 & -28.6 & 0.91 & -27.7 & Bocherens and Drucker (2003) \\
\hline Roe deer & BLW1500 & Bialowieza Forest & Deciduous forest & Collagen & 1994 & -24.0 & -29.1 & 0.91 & -28.2 & Bocherens and Drucker (2003) \\
\hline Roe deer & BLW1600 & Bialowieza Forest & Deciduous forest & Collagen & 1995 & -24.8 & -29.9 & 0.95 & -29.0 & Bocherens and Drucker (2003) \\
\hline Bison & 17270 & Central Saskatchewan & Boreal forest & Hair & 2002 & -25.1 & -28.2 & 1.20 & -27.0 & Urton and Hobson (2005) \\
\hline Bison & 17271 & Central Saskatchewan & Boreal forest & Hair & 2002 & -23.5 & -26.6 & 1.20 & -25.4 & Urton and Hobson (2005) \\
\hline Bison & 17272 & Central Saskatchewan & Boreal forest & Hair & 2002 & -24.7 & -27.8 & 1.20 & -26.6 & Urton and Hobson (2005) \\
\hline Bison & 19969 & Central Saskatchewan & Boreal forest & Hair & 2002 & -25.4 & -28.5 & 1.20 & -27.3 & Urton and Hobson (2005) \\
\hline Bison & 19970 & Central Saskatchewan & Boreal forest & Hair & 2002 & -26.3 & -29.4 & 1.20 & -28.2 & Urton and Hobson (2005) \\
\hline Bison & 19972 & Central Saskatchewan & Boreal forest & Hair & 2002 & -26.6 & -29.7 & 1.20 & -28.5 & Urton and Hobson (2005) \\
\hline Bison & 19973 & Central Saskatchewan & Boreal forest & Hair & 2002 & -25.7 & -28.8 & 1.20 & -27.6 & Urton and Hobson (2005) \\
\hline Bison & 19974 & Central Saskatchewan & Boreal forest & Hair & 2002 & -25.9 & -29.0 & 1.20 & -27.8 & Urton and Hobson (2005) \\
\hline Bison & 19975 & Central Saskatchewan & Boreal forest & Hair & 2002 & -25.9 & -29.0 & 1.20 & -27.8 & Urton and Hobson (2005) \\
\hline Bison & BLW100 & Bialowieza Forest & Deciduous forest & Collagen & 1987 & -23.2 & -28.3 & 0.71 & -27.6 & Bocherens and Drucker (2003) \\
\hline Bison & BLW300 & Bialowieza Forest & Deciduous forest & Collagen & 1998 & -23.0 & -28.1 & 1.05 & -27.1 & Bocherens and Drucker (2003) \\
\hline Bison & BLW400 & Bialowieza Forest & Deciduous forest & Collagen & 1998 & -23.7 & -28.8 & 1.05 & -27.8 & Bocherens and Drucker (2003) \\
\hline Bison & BLW500 & Bialowieza Forest & Deciduous forest & Collagen & 1999 & -24.4 & -29.5 & 1.08 & -28.4 & Bocherens and Drucker (2003) \\
\hline Bison & BLW600 & Bialowieza Forest & Deciduous forest & Collagen & 1999 & -25.6 & -30.7 & 1.08 & -29.6 & Bocherens and Drucker (2003) \\
\hline Bison & ACT400 & Bialowieza Forest & Deciduous forest & Collagen & 1994 & -23.6 & -28.7 & 0.91 & -27.8 & Bocherens and Drucker (2003) \\
\hline
\end{tabular}

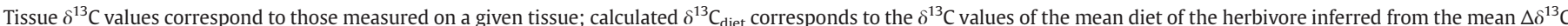

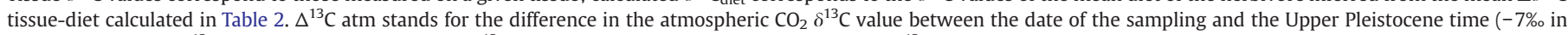

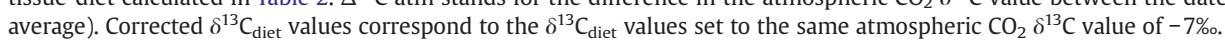

attributions based on archaeological descriptions presented in the original works. In this paper, the term wild bovine refers to bison (Bison sp.) and aurochs (Bos primigenius). When the species was specifically identified by osteological analysis, bison and aurochs provided the same collagen $\delta^{13} \mathrm{C}$ values (Bocherens et al., 2005a,b; and unpublished data). The acquired isotopic data were augmented with data from Bocherens et al. (2005a,b) for the site of Louviers, and from Balasse (1999) for the sites of Cuiry-les-Chaudardes and Maizy. To consider $\delta^{13} \mathrm{C}$ trends through time, we adopted the classical chronological subdivision of Late-Glacial/Early Holocene into chronozones (see Mangerud et al., 1974).

Potential regional effects on herbivore $\delta^{13} \mathrm{C}$ values were examined using samples of red deer from threes sites in the Jura and four sites in the northern Alps. Both regions, although geographically close, exhibit differences in faunal succession during the Late-Glacial/Early Holocene transition (Bridault and Chaix, 2002). AMS radiocarbon dating was performed at the Radiocarbon Laboratory of the University of Groningen (The Netherlands) on each sample of collagen red deer, which allowed us to organise our results chronologically. The radiocarbon dates have been calibrated to cal years BP using the IntCal04 calibration dataset (Reimer et al., 2004) with the software calib.5.0.1.

Isotopic data from modern caribou from Queen Maud Gulf were measured on hair, after a cleaning protocol following Urton and
Hobson (2005). Isotopic data from all ancient mammals were performed on skeletal collagen. Collagen extraction on modern and ancient material was performed following Bocherens et al. (1997). The reliability of the isotope values were checked using the carbon and nitrogen elemental composition of the collagen $\left(\mathrm{C}_{\text {coll }}, \mathrm{N}_{\text {coll }}\right)$. Indeed, isotopically well-preserved collagen exhibit $\mathrm{C} / \mathrm{N}_{\text {coll }}$ ratios ranging from 2.9 to 3.6 and percentage of $\mathrm{C}_{\text {coll }}$ and $\mathrm{N}_{\text {coll }}$ higher than $8 \%$ and $3 \%$, respectively (Ambrose, 1990). Elemental composition of the extracted collagen was measured on a CHN-elemental analyzer (Carlo-Erba NA15000 and Eurovector) coupled to a VG-Optima continuous-flow ratio mass spectrometer used for ${ }^{13} \mathrm{C} /{ }^{12} \mathrm{C}$ measurements at the Earth Science Department of University P. et M. Curie (Paris, France) and in the Institut des Sciences de l'Evolution (Université Montpellier 2, France). Stable isotope ratios are expressed as $\delta^{13} \mathrm{C}$ values relative to the international standard VPDB, with an analytical error of $0.1 \%$.

\section{Results}

Values of $\delta^{13} \mathrm{C}_{\text {diet }}$ calculated from modern large herbivores varied between $-30.4 \%$ 。 to $-23.6 \%$ o with an inter-individual variation within a given population that does not exceed $3.1 \%$, as measured on bison from Central Saskatchewan (Table 3; Fig. 3). Reindeer from Canadian arctic tundra and Siberian open taiga provided some of the highest 


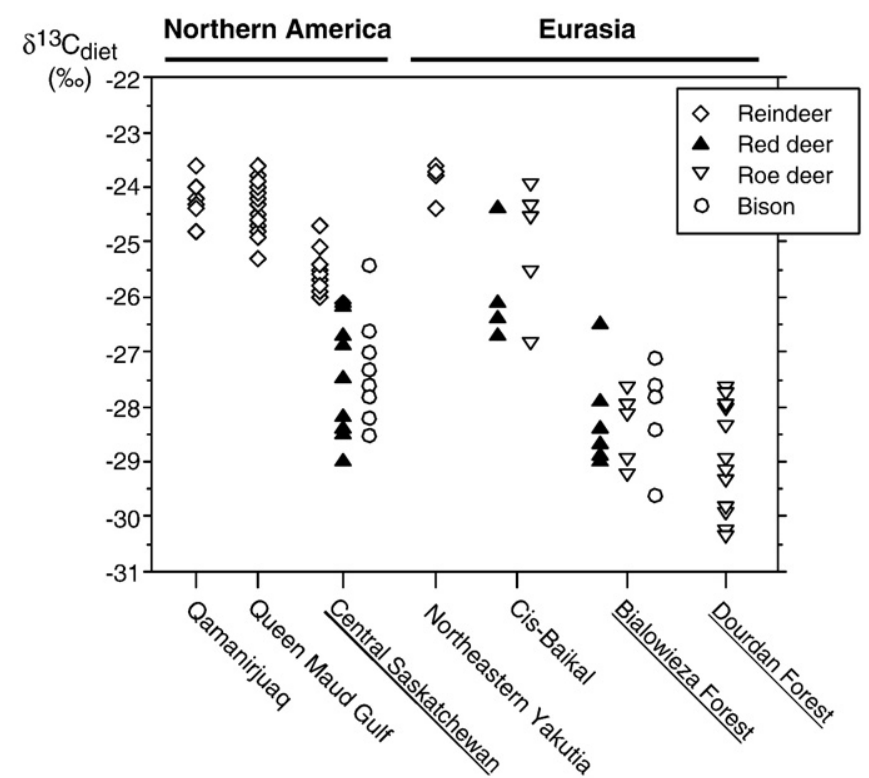

Fig. 3. Review of $\delta^{13} C_{\text {diet }}$ values of herbivores from modern ecosystems from North America and Eurasia. Closed-canopy environments are underlined.

$\delta^{13} \mathrm{C}_{\text {diet }}$ values, while roe deer from temperate forests in Europe exhibited some of the lowest $\delta^{13} C_{\text {diet }}$ values.

For a given species, the populations dwelling in a closed canopy forest always exhibited significantly lower $\delta^{13} \mathrm{C}$ values than populations dwelling in a more open environment. This is observed when comparing woodland caribou on one hand with barren-ground caribou and open taiga reindeer on the other hand, red deer from boreal or temperate closed canopy forests with red deer from open forest-steppe, roe deer from temperate closed canopy forests with roe deer from steppe and forest-steppe (Fig. 3; Table 4). In contrast, when dealing with a similar type of habitat, open or closed, no significant difference is observed despite clear differences in the geographical origin, the climatic conditions, and the floristic composition. For instance, roe deer from the temperate forest from Dourdan, dominated by beech and roe deer from Bialowieza forest, dominated by oak, lime and hornbeam, exhibit equivalent $\delta^{13} \mathrm{C}$ values despite a difference of $6{ }^{\circ} \mathrm{C}$ between the average annual temperatures in both locations (Table 1 ). Moreover, the $\delta^{13} \mathrm{C}$ values of a given taxon in closed canopy forest of boreal or temperate type are not significantly different, as for bison and red deer from Central Saskatchewan and Bialowieza Forest (Fig. 3; Table 4). In open environments, barrenground caribou from both Canadian populations and open taiga reindeer from Yakutia also exhibit equivalent $\delta^{13} \mathrm{C}$ values (Table 4).
These results are not affected by the tissue used to calculate the $\delta^{13} \mathrm{C}$ value of the diet, as well illustrated by barren-ground caribou from Queen Maud Gulf or Qamanirjuaq herds, which provided equivalent $\delta^{13} \mathrm{C}_{\text {diet }}$ although hair or collagen were sampled.

During the Late-Glacial/Early Holocene transition, the $\delta^{13} C_{\text {diet }}$ values of cervids and large bovines from Paris Basin exhibited a significant decrease (Table 5; Fig. 4); however, this decrease differed among species. Indeed, depletion of $\delta^{13} C_{\text {diet }}$ values through the transition was more pronounced for wild bovine $(-3.3 \%$ ) than for red deer $\left(-2.2 \%\right.$ ) and roe deer $\left(-2.7 \%\right.$ o). As a result, $\delta^{13} \mathrm{C}_{\text {diet }}$ values of wild bovine were comparable to those of red deer and roe deer during the Boreal/early Atlantic transition (ca. 9100-8000 years cal BP), whereas they were significantly higher (Mann-Whitney U-test, $U=21.0, p<0.005$ ) during the Bölling time (ca. 14,700-13,900 years cal BP). The $\delta^{13} \mathrm{C}_{\text {diet }}$ values of roe deer reached a minimum during the early Atlantic (ca. 9000-7000 years cal BP) after which a small increase was observed. The $\delta^{13} C_{\text {diet }}$ values of red deer and wild bovine changed slightly at the end of the Atlantic, towards less depleted values for red deer and more depleted values for wild bovine. Domestic cattle present similar $\delta^{13} \mathrm{C}_{\text {diet }}$ values as wild bovine during the early Atlantic. In the late Atlantic, the $\delta^{13} C_{\text {diet }}$ values of domestic cattle changed towards significantly less-depleted values than wild bovine (Mann-Whitney $U$-test, $p<0.0001, U=4.0, n>10$ ).

Dated specimens of red deer from Jura and northern Alps exhibited a general trend of decreasing $\delta^{13} \mathrm{C}_{\text {diet }}$ values over the Late-Glacial/ Early Holocene transition (Tables 6 and 7; Fig. 5). Red deer $\delta^{13} \mathrm{C}_{\text {diet }}$ values ranged between $-24 \%$ and $-27 \%$ o during the Bölling/Alleröd period for both Jura and northern Alps areas. After the Younger Dryas, red deer $\delta^{13} C_{\text {diet }}$ values ranged from $-27 \%$ o to $-29.1 \%$ o in the Jura, while red deer from the northern Alps had $\delta^{13} \mathrm{C}_{\text {diet }}$ values similar to those observed before the Younger Dryas. As a result, the Late-Glacial/ Early Holocene trend in red deer towards about 3\% lower $\delta^{13} \mathrm{C}_{\text {diet }}$ values is evidenced in the Jura, but not in the northern Alps. This confirms that, in addition to global change, local factors can account for changes in $\delta^{13} C_{\text {diet }}$ of large herbivores at the Early Holocene.

\section{Discussion}

\subsection{Modern ecosystems}

For every species considered, the populations dwelling under closed canopy habitats exhibit significantly lower $\delta^{13} \mathrm{C}$ values than those dwelling under more open conditions. This was not simply linked to shift from grazing to browsing with increasing forest cover, since it occurred for a specialist feeder like roe deer as well as for a generalist one like red deer. This was not surprising since vascular plants show depleted ${ }^{13} \mathrm{C}$ abundances under closed canopy cover compared to more open conditions. A good illustration of this is the comparison of $\delta^{13} \mathrm{C}$ values from Brooks et al. (1997) for boreal forest

Table 4

Statistical comparisons (Mann-Whitney) of $\delta^{13} \mathrm{C}$ values obtained on populations from a given species

\begin{tabular}{|c|c|c|c|c|}
\hline Taxon & Tissue & Environments ( $\mathrm{n}$ ) & Type & Statistical comparison \\
\hline Rangifer tarandus & $\mathrm{C} / \mathrm{H}$ & Qamanirjuaq (arctic tundra) ( $n=6) /$ Queen Maud Gulf (arctic tundra) $(n=24)$ & $\mathrm{O} / \mathrm{O}$ & $p=0.32, U=53.0$ \\
\hline Rangifer tarandus & $\mathrm{C} / \mathrm{C}$ & Qamanirjuaq (arctic tundra) $(n=6) /$ NE Yakutia (open taiga) $(n=5)$ & $0 / 0$ & $p=0.52, U=11.5$ \\
\hline Rangifer tarandus & $\mathrm{H} / \mathrm{C}$ & Queen Maud Gulf (arctic tundra) $(n=24) /$ NE Yakutia (open taiga) $(n=5)$ & $0 / 0$ & $p=0.13, U=38.5$ \\
\hline Cervus elaphus & $\mathrm{H} / \mathrm{C}$ & Prince Albert Natl. Park (boreal forest) $(n=9) /$ Bialowieza Natl. Park (temperate forest) $(n=6)$ & $\mathrm{C} / \mathrm{C}$ & $p=0.19, U=16.0$ \\
\hline Capreolus capreolus & $\mathrm{C} / \mathrm{C}$ & Dourdan forest (temperate forest) $(n=18) /$ Bialowieza Natl. Park (temperate forest) $(n=5)$ & $\mathrm{C} / \mathrm{C}$ & $p=0.19, U=27.5$ \\
\hline Bison sp. & $\mathrm{H} / \mathrm{C}$ & Prince Albert Natl. Park (boreal forest) $(n=9)$ / Bialowieza Natl. Park (temperate forest) $(n=6)$ & $\mathrm{C} / \mathrm{C}$ & $p=0.26, U=17.5$ \\
\hline Rangifer tarandus & $\mathrm{C}+\mathrm{H} / \mathrm{H}$ & Qamanirjuaq +Queen Maud Gulf (arctic tundra) $(n=30) /$ Prince Albert Natl. Park (boreal forest) $(n=14)$ & $\mathrm{O} / \mathrm{C}$ & $p<0.0001, U=10.5$ \\
\hline Rangifer tarandus & $\mathrm{H} / \mathrm{H}$ & Queen Maud Gulf (arctic tundra) ( $n=24)$ / Prince Albert Natl. Park (boreal forest) $(n=14)$ & $\mathrm{O} / \mathrm{C}$ & $P<0.0001, U=9.5$ \\
\hline Cervus elaphus & $\mathrm{C} / \mathrm{C}$ & Cis-Baikal (forest-steppe) $(n=6) /$ Bialowieza Natl. Park (temperate forest) $(n=6)$ & $\mathrm{O} / \mathrm{C}$ & $p=0.02, U=1.0$ \\
\hline Cervus elaphus & $\mathrm{C} / \mathrm{H}$ & Cis-Baikal (forest-steppe) $(n=6) /$ Prince Albert Natl. Park (boreal forest) $(n=9)$ & $\mathrm{O} / \mathrm{C}$ & $p=0.02, U=6.0$ \\
\hline Capreolus capreolus & $\mathrm{C} / \mathrm{C}$ & Cis-Baikal (forest-steppe) $(n=6) /$ Dourdan forest + Bialowieza Natl. Park (temperate forest) $(n=23)$ & $\mathrm{O} / \mathrm{C}$ & $p=0.0002, U=0.0$ \\
\hline
\end{tabular}

Statistically significant results are shown in bold.

$\mathrm{C}$ and $\mathrm{H}$ correspond to collagen and hair. $\mathrm{O}$ and $\mathrm{C}$ correspond to open and closed-canopy environments. 
Table 5

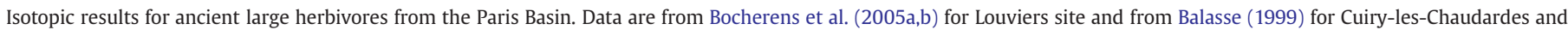
Maizy sites

\begin{tabular}{|c|c|c|c|c|c|c|c|c|c|c|}
\hline Species & Lab $n^{\circ}$ & Site & Sample & Excavation $n^{\circ}$ & Chronozone & $\begin{array}{l}\text { Ccoll } \\
(\%)\end{array}$ & $\begin{array}{l}\text { Ncoll } \\
(\%)\end{array}$ & C/Ncoll & $\begin{array}{l}\delta^{13} \mathrm{C}_{\text {coll }} \\
(\%)\end{array}$ & $\begin{array}{l}\delta^{13} \mathrm{C}_{\text {diet }} \\
(\%)\end{array}$ \\
\hline Red deer & BRG2800 & Bois-Ragot (layer 5) & Left tibia & BR 5 I5 736 & Bölling & 24.6 & 8.7 & 3.3 & -20.8 & -25.9 \\
\hline Red deer & BRG2900 & Bois-Ragot (layer 5) & Right radius & BR 5 I1 434 & Bölling & 19.5 & 7.7 & 2.9 & -20.0 & -25.1 \\
\hline Red deer & BRG3000 & Bois-Ragot (layer 5) & Left humerus & BR 5 H2 1096 & Bölling & 21.5 & 7.4 & 3.4 & -21.0 & -26.1 \\
\hline Red deer & BRG4200 & Bois-Ragot (layer 4) & Left scapula & BR 4 I7 252 & Bölling & 33.2 & 12.3 & 3.1 & -20.6 & -25.7 \\
\hline Red deer & BRG4300 & Bois-Ragot (layer 4) & Left scapula & BR 4 J1 67 & Bölling & 30.1 & 11.4 & 3.1 & -21.0 & -26.1 \\
\hline Red deer & BRG4400 & Bois-Ragot (layer 4) & Right metacarpal & BR 4 H2 249 & Bölling & 36.8 & 13.3 & 3.2 & -19.7 & -24.8 \\
\hline Red deer & BRG4500 & Bois-Ragot (layer 4) & Right metacarpal & BR 4 H6 1 & Bölling & 30.9 & 12.0 & 3.0 & -20.5 & -25.6 \\
\hline Red deer & BRG4600 & Bois-Ragot (layer 4) & Right metatarsal & BR 4 H2 163 & Bölling & 37.5 & 13.8 & 3.2 & -20.4 & -25.5 \\
\hline Red deer & BRG4700 & Bois-Ragot (layer 4) & Right metatarsal & BR 4 J7 68 & Bölling & 32.4 & 12.3 & 3.1 & -20.1 & -25.2 \\
\hline Red deer & BRG4800 & Bois-Ragot (layer 4) & Right metatarsal & BR 4 I1 195 & Bölling & 24.9 & 9.7 & 3.0 & -21.3 & -26.4 \\
\hline Red deer & BRG5900 & Bois-Ragot (layer 3) & Metatarsal & BR $3 \mathrm{~J} 3$ & Bölling & 35.4 & 12.8 & 3.2 & -20.9 & -26.0 \\
\hline Red deer & BRG6000 & Bois-Ragot (layer 3) & Radius & BR 3 H7-7 & Bölling & 38.8 & 14.7 & 3.1 & -20.3 & -25.4 \\
\hline Red deer & CLS1000 & Le Closeau (locus 46) & Left tibia & LC97IFP3S6L46 E268 139 & Bölling & 38.0 & 13.8 & 3.2 & -20.1 & -25.2 \\
\hline Red deer & CLS1100 & Le Closeau (locus 46) & Left tibia & LC97IFP3S6L46 E270 67 & Bölling & 35.4 & 12.8 & 3.2 & -20.2 & -25.3 \\
\hline Red deer & CLS1200-3 & Le Closeau (locus 46) & Left tibia & LC97IFP3S6L46 D268 280 & Bölling & 40.5 & 14.7 & 3.2 & -20.4 & -25.5 \\
\hline Red deer & CLS1300 & Le Closeau (locus 46) & Right tibia & LC97IFP3S6L46 E267 13 & Bölling & 38.1 & 13.9 & 3.2 & -20.5 & -25.6 \\
\hline Red deer & CLS1400-1 & Le Closeau (locus 46) & Right tibia & LC97IFP3S6L46 D267 82 & Bölling & 39.1 & 14.3 & 3.2 & -20.2 & -25.3 \\
\hline Red deer & CLS1500 & Le Closeau (locus 46) & Right tibia & LC97IFP3S6L46 Z266 15 & Bölling & 39.1 & 14.2 & 3.2 & -20.5 & -25.6 \\
\hline Red deer & N0100 & Noyen-sur-Seine (layer 9) & Right radius & 84 XVI C150-8 & Boreal/Atlantic & 41.6 & 15.1 & 3.2 & -22.8 & -27.9 \\
\hline Red deer & NO300 & Noyen-sur-Seine (layer 7d) & Left humerus & 84 XV Z153-7 & Boreal/Atlantic & 39.7 & 14.2 & 3.3 & -22.7 & -27.8 \\
\hline Red deer & NO400 & Noyen-sur-Seine (layer 9) & Left humerus & 84 XVI H140-6 & Boreal/Atlantic & 42.0 & 15.8 & 3.0 & -22.3 & -27.4 \\
\hline Red deer & NO600 & Noyen-sur-Seine (layer 9) & Left radius & 84 XVI D144-6 & Boreal/Atlantic & 41.6 & 14.8 & 3.3 & -23.1 & -28.2 \\
\hline Red deer & NO4800 & Noyen-sur-Seine (layer 9) & Left metacapal & 84 XVI E137-12 & Boreal/Atlantic & 41.8 & 15.1 & 3.2 & -22.5 & -27.6 \\
\hline Red deer & NO5000 & Noyen-sur-Seine (layer 9) & Left metacapal & 84 XVI D143-15 & Boreal/Atlantic & 42.5 & 15.6 & 3.2 & -22.5 & -27.6 \\
\hline Red deer & CCF cer 1 & Cuiry-les-Chaudardes & n.g. & n.g. & Early Atlantic & 40.2 & 14.7 & 3.2 & -22.8 & -27.9 \\
\hline Red deer & CCF cer 2 & Cuiry-les-Chaudardes & n.g. & n.g. & Early Atlantic & 39.9 & 14.6 & 3.2 & -22.7 & -27.8 \\
\hline Red deer & CCF cer 3 & Cuiry-les-Chaudardes & n.g. & n.g. & Early Atlantic & 39.3 & 14.4 & 3.2 & -23.4 & -28.5 \\
\hline Red deer & CCF cer 4 & Cuiry-les-Chaudardes & n.g. & n.g. & Early Atlantic & 32.4 & 11.8 & 3.2 & -20.6 & -25.7 \\
\hline Red deer & CCF cer 5 & Cuiry-les-Chaudardes & n.g. & n.g. & Early Atlantic & 41.3 & 15.2 & 3.2 & -24.0 & -29.1 \\
\hline Red deer & MGA cer 1 & Maizy & n.g. & n.g. & Late Atlantic & 40.5 & 14.8 & 3.2 & -20.2 & -25.3 \\
\hline Red deer & MGA cer 2 & Maizy & n.g. & n.g. & Late Atlantic & 41.8 & 15.4 & 3.2 & -22.7 & -27.8 \\
\hline Red deer & MGA cer 3 & Maizy & n.g. & n.g. & Late Atlantic & 36.9 & 13.5 & 3.2 & -22.2 & -27.3 \\
\hline Red deer & MGA cer 4 & Maizy & n.g. & n.g. & Late Atlantic & 40.8 & 15.0 & 3.2 & -22.9 & -28.0 \\
\hline Red deer & B2601 & Bercy & Humerus & QS, L XVI,25 c21 & Late Atlantic & 39.4 & 13.3 & 3.5 & -23.0 & -28.1 \\
\hline Red deer & B5000 & Bercy & Radius & QS, M XV,8 c2 & Late Atlantic & 39.5 & 14.6 & 3.2 & -23.3 & -28.4 \\
\hline Red deer & B5400 & Bercy & Metapodal & $\mathrm{QS}, \mathrm{M} X \mathrm{XV}, 11$ & Late Atlantic & 39.7 & 14.6 & 3.2 & -21.6 & -26.7 \\
\hline Red deer & B5200 & Bercy & Radius & $\mathrm{QS}, \mathrm{M} \mathrm{XV}, 14 \mathrm{c} 2$ & Late Atlantic & 40.9 & 15.0 & 3.2 & -23.5 & -28.6 \\
\hline Red deer & B5100 & Bercy & Radius & QS, M XV,20 c2n3 & Late Atlantic & 42.1 & 15.4 & 3.2 & -22.3 & -27.4 \\
\hline Red deer & B5300 & Bercy & Humerus & $\mathrm{QS}, \mathrm{M} \mathrm{XV}, 20 \mathrm{c} 2 \mathrm{n} 4$ & Late Atlantic & 37.5 & 13.7 & 3.2 & -22.5 & -27.6 \\
\hline Red deer & B4800 & Bercy & Tibia & $\mathrm{QS}, \mathrm{M}$ XV,14 c2n4 & Late Atlantic & 41.5 & 15.2 & 3.2 & -21.4 & -26.5 \\
\hline Red deer & Lo3000 & Louviers & Left humerus & A c18 24,84 & Late Atlantic & 44.2 & 15.1 & 3.4 & -22.0 & -27.1 \\
\hline Red deer & Lo3100 & Louviers & Right radius & A c18 24,83 & Late Atlantic & 44.7 & 15.5 & 3.4 & -22.4 & -27.5 \\
\hline Red deer & Lo3200 & Louviers & Right radius & A c18 24,82 & Late Atlantic & 44.5 & 15.4 & 3.4 & -22.1 & -27.2 \\
\hline Red deer & Lo3300 & Louviers & Left humerus & A c16 29,92 & Late Atlantic & 44.7 & 15.2 & 3.4 & -22.2 & -27.3 \\
\hline Red deer & Lo3400 & Louviers & Right tibia & A c16 34,80 & Late Atlantic & 44.1 & 14.8 & 3.5 & -22.9 & -28.0 \\
\hline Roe deer & BRG700 & Bois-Ragot (layer 6) & Metatarsal & BR 6 G4 559 & Bölling & 35.6 & 13.3 & 3.1 & -20.2 & -25.3 \\
\hline Roe deer & BRG800 & Bois-Ragot (layer 6) & Femur & BR 6 G4 508 & Bölling & 28.1 & 11.2 & 2.9 & -20.9 & -26.0 \\
\hline Roe deer & BRG5000 & Bois-Ragot (layer 4) & Metatarsal & BR 4 J2 173 & Bölling & 36.8 & 13.9 & 3.1 & -20.4 & -25.5 \\
\hline Roe deer & BRG5100 & Bois-Ragot (layer 4) & Metatarsal & BR 4 K5 79 & Bölling & 18.1 & 6.9 & 3.1 & -19.0 & -24.1 \\
\hline Roe deer & BRG6300 & Bois-Ragot (layer 3) & Metacarpal & BR 3 J5-110 & Bölling & 39.2 & 14.9 & 3.1 & -20.5 & -25.6 \\
\hline Roe deer & BRG6400 & Bois-Ragot (layer 3) & Metacarpal & BR 3 H1-26 & Bölling & 37.4 & 13.9 & 3.1 & -20.9 & -26.0 \\
\hline Roe deer & NO2100 & Noyen-sur-Seine (layer 9) & Phalanx I & 83 XVI E140 & Boreal/Atlantic & 42.0 & 15.5 & 3.2 & -22.7 & -27.8 \\
\hline Roe deer & NO2200 & Noyen-sur-Seine (layer 9) & Phalanx I & 83 XVI E140 & Boreal/Atlantic & 42.3 & 15.5 & 3.2 & -22.4 & -27.5 \\
\hline Roe deer & NO2400 & Noyen-sur-Seine (layer 9) & Phalanx I & 84 XVI Y151-36 & Boreal/Atlantic & 41.9 & 15.2 & 3.3 & -23.1 & -28.2 \\
\hline Roe deer & N05300 & Noyen-sur-Seine (layer 9) & Left femur & 84 XV Z151-36 & Boreal/Atlantic & 41.6 & 15.1 & 3.2 & -22.9 & -28.0 \\
\hline Roe deer & NO5400 & Noyen-sur-Seine (layer 9) & Left tibia & 84 XVI G142-6 & Boreal/Atlantic & 42.2 & 15.5 & 3.2 & -22.8 & -27.9 \\
\hline Roe deer & NO5600 & Noyen-sur-Seine (layer 9) & Phalanx I & 84 XVI H132-38 & Boreal/Atlantic & 42.7 & 15.7 & 3.2 & -23.2 & -28.3 \\
\hline Roe deer & NO5500 & Noyen-sur-Seine (layer 9) & Left tibia & 84 XVI E141-2 & Boreal/Atlantic & 42.8 & 15.8 & 3.2 & -23.8 & -28.9 \\
\hline Roe deer & CCF ch 1 & Cuiry-les-Chaudardes & n.g. & n.g. & Early Atlantic & 39.6 & 14.4 & 3.2 & -23.4 & -28.5 \\
\hline Roe deer & CCF ch 2 & Cuiry-les-Chaudardes & n.g. & n.g. & Early Atlantic & 41.4 & 15.1 & 3.2 & -23.7 & -28.8 \\
\hline Roe deer & CCF ch 3 & Cuiry-les-Chaudardes & n.g. & n.g. & Early Atlantic & 39.8 & 14.6 & 3.2 & -22.8 & -27.9 \\
\hline Roe deer & CCF ch 4 & Cuiry-les-Chaudardes & n.g. & n.g. & Early Atlantic & 41.5 & 15.2 & 3.2 & -24.5 & -29.6 \\
\hline Roe deer & CCF ch 5 & Cuiry-les-Chaudardes & n.g. & n.g. & Early Atlantic & 40.4 & 14.7 & 3.2 & -24.4 & -29.5 \\
\hline Roe deer & Lo4700 & Louviers & Skull & c12 42,83 & Late Atlantic & 40.9 & 13.6 & 3.5 & -23.9 & -29.0 \\
\hline Roe deer & B4200 & Bercy & Jawbone & QS, N XIII,13 c,15 st,37 & Late Atlantic & 38.4 & 13.7 & 3.3 & -22.3 & -27.4 \\
\hline Roe deer & B4300 & Bercy & Scapula & QS, M XX,11 c,21 & Late Atlantic & 39.7 & 14.3 & 3.2 & -22.2 & -27.3 \\
\hline Roe deer & B4400 & Bercy & Scapula & $\mathrm{QS}, \mathrm{M} X \mathrm{XV}, 15$ c,2n3 & Late Atlantic & 41.2 & 15.2 & 3.2 & -23.2 & -28.3 \\
\hline Roe deer & B4500 & Bercy & Radius and ulna & $\mathrm{QS}, \mathrm{M} \mathrm{XV}, 14$ & Late Atlantic & 41.1 & 15.1 & 3.2 & -22.8 & -27.9 \\
\hline Wild bovine & BRG1400 & Bois-Ragot (layer 6) & Inf M3 & BR 6 H4 242 & Bölling & 41.8 & 15.3 & 3.2 & -19.5 & -24.6 \\
\hline Wild bovine & BRG3100 & Bois-Ragot (layer 5) & Humerus G & BR 5 J4 819 & Bölling & 41.0 & 15.2 & 3.2 & -19.5 & -24.6 \\
\hline Wild bovine & BRG3200 & Bois-Ragot (layer 5) & Humerus G & BR 5 F2 147 & Bölling & 39.2 & 15.0 & 3.1 & -19.9 & -25.0 \\
\hline Wild bovine & BRG3700 & Bois-Ragot (layer 4) & Radius D & BR 4 I5 608 & Bölling & 39.0 & 14.8 & 3.1 & -19.4 & -24.5 \\
\hline Wild bovine & BRG3800 & Bois-Ragot (layer 4) & Radius D & BR 4 J4 706 & Bölling & 35.5 & 13.8 & 3.0 & -19.8 & -24.9 \\
\hline
\end{tabular}


Table 5 (continued)

\begin{tabular}{|c|c|c|c|c|c|c|c|c|c|c|}
\hline Species & $\operatorname{Lab}^{\circ}$ & Site & Sample & Excavation $\mathrm{n}^{\circ}$ & Chronozone & $\begin{array}{l}\text { Ccoll } \\
(\%)\end{array}$ & $\begin{array}{l}\text { Ncoll } \\
(\%)\end{array}$ & C/Ncoll & $\begin{array}{l}\delta^{13} \mathrm{C}_{\text {coll }} \\
(\%)\end{array}$ & $\begin{array}{l}\delta^{13} C_{\text {diet }} \\
(\%) \\
\end{array}$ \\
\hline Wild bovine & BRG3900 & Bois-Ragot (layer 4) & Phalanx I & BR 4 J4 591 & Bölling & 34.9 & 13.3 & 3.1 & -20.0 & -25.1 \\
\hline Wild bovine & BRG4000 & Bois-Ragot (layer 4) & Humerus & BR 4 J3 565 & Bölling & 35.3 & 13.6 & 3.0 & -19.1 & -24.2 \\
\hline Wild bovine & BRG4100 & Bois-Ragot (layer 4) & Humerus & BR 4 J3 677 & Bölling & 35.5 & 13.5 & 3.1 & -20.4 & -25.5 \\
\hline Wild bovine & N01600 & Noyen-sur-Seine (layer 9) & Phalanx III & 84 XVI C150-8 & Boreal/Atlantic & 41.4 & 14.8 & 3.3 & -23.5 & -28.6 \\
\hline Wild bovine & N01900 & Noyen-sur-Seine (layer 9) & Metapodal & 84 XVI C149-6 & Boreal/Atlantic & 41.7 & 15.2 & 3.2 & -23.4 & -28.5 \\
\hline Wild bovine & NO2000 & Noyen-sur-Seine (layer 9) & Metatarsal & 84 XV TZ153-9 & Boreal/Atlantic & 41.5 & 15.1 & 3.2 & -22.8 & -27.9 \\
\hline Wild bovine & NO5100 & Noyen-sur-Seine (layer 9) & Left metatarsal & 84 XVI G144-4 & Boreal/Atlantic & 42.5 & 15.4 & 3.2 & -22.9 & -28.0 \\
\hline Wild bovine & NO5200 & Noyen-sur-Seine (layer 9) & Left tibia & 84 XVI B148-14 & Boreal/Atlantic & 41.7 & 15.2 & 3.2 & -22.6 & -27.7 \\
\hline Wild bovine & CCF aur 1 & Cuiry-les-Chaudardes & n.g. & n.g. & Early Atlantic & 32.0 & 11.6 & 3.2 & -22.6 & -27.7 \\
\hline Wild bovine & CCF aur 2 & Cuiry-les-Chaudardes & n.g. & n.g. & Early Atlantic & 31.1 & 11.4 & 3.2 & -23.0 & -28.1 \\
\hline Wild bovine & CCF aur 3 & Cuiry-les-Chaudardes & n.g. & n.g. & Early Atlantic & 28.1 & 10.3 & 3.2 & -23.5 & -28.6 \\
\hline Wild bovine & CCF aur 4 & Cuiry-les-Chaudardes & n.g. & n.g. & Early Atlantic & 37.0 & 13.6 & 3.2 & -23.6 & -28.7 \\
\hline Wild bovine & CCF aur 5 & Cuiry-les-Chaudardes & n.g. & n.g. & Early Atlantic & 42.5 & 15.6 & 3.2 & -23.5 & -28.6 \\
\hline Wild bovine & CCF aur 6 & Cuiry-les-Chaudardes & n.g. & n.g. & Early Atlantic & 41.1 & 15.1 & 3.2 & -22.7 & -27.8 \\
\hline Wild bovine & MGA aur 1 & Maizy & n.g. & n.g. & Late Atlantic & 40.3 & 14.8 & 3.2 & -24.0 & -29.1 \\
\hline Wild bovine & MGA aur 2 & Maizy & n.g. & n.g. & Late Atlantic & 42.4 & 15.5 & 3.2 & -23.8 & -28.9 \\
\hline Wild bovine & MGA aur 3 & Maizy & n.g. & n.g. & Late Atlantic & 36.9 & 13.6 & 3.2 & -24.5 & -29.6 \\
\hline Wild bovine & B2001 & Bercy & Radius & QS, L XV, 15 c21 & Late Atlantic & 38.3 & 12.9 & 3.5 & -23.6 & -28.7 \\
\hline Wild bovine & B2201C & Bercy & Tibia & QS, L XII,13 c21 & Late Atlantic & 43.0 & 15.4 & 3.3 & -24.0 & -29.1 \\
\hline Wild bovine & B5500 & Bercy & Sacrum & $\mathrm{QS}, \mathrm{M} \mathrm{XV}, 8 \mathrm{c} 2 \mathrm{n} 4$ & Late Atlantic & 41.7 & 15.3 & 3.2 & -23.4 & -28.5 \\
\hline Wild bovine & B5600C & Bercy & Metatarsal & $\mathrm{QS}, \mathrm{L} \mathrm{XV}, 24 \mathrm{c} 21$ & Late Atlantic & 42.5 & 15.3 & 3.2 & -23.4 & -28.5 \\
\hline Wild bovine & $\mathrm{B} 6100 \mathrm{C}$ & Bercy & Humerus & $\mathrm{QS}, \mathrm{L} X \mathrm{XV}, 12$ c21 & Late Atlantic & 42.5 & 15.3 & 3.2 & -23.7 & -28.8 \\
\hline Wild bovine & B6200C & Bercy & Radius and ulna & QS, K XVIII,25 c21 & Late Atlantic & 43.5 & 15.7 & 3.2 & -23.1 & -28.2 \\
\hline Wild bovine & Lo3600 & Louviers & Phalanx I & A c18 34,80 & Late Atlantic & 44.2 & 15.3 & 3.4 & -23.6 & -28.7 \\
\hline Wild bovine & Lo3700 & Louviers & Phalanx I & A c18 48,81 & Late Atlantic & 43.8 & 15.1 & 3.4 & -22.8 & -27.9 \\
\hline Wild bovine & Lo3900 & Louviers & Humerus & A c18 53,80 & Late Atlantic & 43.8 & 15.0 & 3.4 & -23.0 & -28.1 \\
\hline Wild bovine & Lo4000 & Louviers & Right metacarpal & A c16 31,82 & Late Atlantic & 43.9 & 15.1 & 3.4 & -22.7 & -27.8 \\
\hline Cattle & CCF303 & Cuiry-les-Chaudardes & n.g. & n.g. & Early Atlantic & 41.8 & 15.2 & 3.2 & -23.2 & -28.3 \\
\hline Cattle & CCF502 & Cuiry-les-Chaudardes & n.g. & n.g. & Early Atlantic & 42.7 & 15.5 & 3.2 & -23.5 & -28.6 \\
\hline Cattle & CCF803 & Cuiry-les-Chaudardes & n.g. & n.g. & Early Atlantic & 42.6 & 15.7 & 3.2 & -22.8 & -27.9 \\
\hline Cattle & CCF1302 & Cuiry-les-Chaudardes & n.g. & n.g. & Early Atlantic & 42.2 & 15.4 & 3.2 & -22.2 & -27.3 \\
\hline Cattle & CCF1403 & Cuiry-les-Chaudardes & n.g. & n.g. & Early Atlantic & 41.0 & 14.9 & 3.2 & -23.0 & -28.1 \\
\hline Cattle & CCF1503 & Cuiry-les-Chaudardes & n.g. & n.g. & Early Atlantic & 41.5 & 15.1 & 3.2 & -22.5 & -27.6 \\
\hline Cattle & CCF1603 & Cuiry-les-Chaudardes & n.g. & n.g. & Early Atlantic & 40.1 & 14.8 & 3.2 & -23.5 & -28.6 \\
\hline Cattle & CCF1701 & Cuiry-les-Chaudardes & n.g. & n.g. & Early Atlantic & 40.6 & 14.9 & 3.2 & -23.4 & -28.5 \\
\hline Cattle & MGA300 & Maizy & n.g. & n.g. & Late Atlantic & 42.7 & 15.6 & 3.2 & -22.3 & -27.4 \\
\hline Cattle & MGA500 & Maizy & n.g. & n.g. & Late Atlantic & 40.9 & 15.1 & 3.2 & -22.2 & -27.3 \\
\hline Cattle & MGA603 & Maizy & n.g. & n.g. & Late Atlantic & 42.9 & 15.7 & 3.2 & -21.9 & -27.0 \\
\hline Cattle & MGA703 & Maizy & n.g. & n.g. & Late Atlantic & 30.2 & 10.8 & 3.3 & -21.6 & -26.7 \\
\hline Cattle & B8600 & Bercy & Metapodal & QS, M XV,10 c2n4 & Late Atlantic & 41.5 & 15.3 & 3.2 & -21.6 & -26.7 \\
\hline Cattle & B8500 & Bercy & Tibia & $\mathrm{QS}, \mathrm{M} X \mathrm{XV}, 14 \mathrm{c} 2 \mathrm{n} 4$ & Late Atlantic & 40.9 & 15.0 & 3.2 & -22.7 & -27.8 \\
\hline Cattle & B7400 & Bercy & Radius & QS, M XVI,22 c22 & Late Atlantic & 40.3 & 14.9 & 3.2 & -21.3 & -26.4 \\
\hline Cattle & B7500 & Bercy & Radius & QS, M XVI,22 c21 & Late Atlantic & 40.1 & 14.8 & 3.2 & -21.9 & -27.0 \\
\hline Cattle & B8700 & Bercy & Metapodal & $\mathrm{QS}, \mathrm{M} X \mathrm{XV}, 8 \mathrm{c} 2 \mathrm{n} 4$ & Late Atlantic & 41.8 & 15.4 & 3.2 & -22.0 & -27.1 \\
\hline Cattle & B8800 & Bercy & Metapodal & $\mathrm{QS}, \mathrm{M} X \mathrm{XV}, 18 \mathrm{c} 2 \mathrm{n} 4$ & Late Atlantic & 41.6 & 14.9 & 3.3 & -21.8 & -26.9 \\
\hline Cattle & B8900 & Bercy & Tibia & $\mathrm{QS}, \mathrm{M} X \mathrm{XVI}, 3 \mathrm{c1}$ & Late Atlantic & 41.6 & 14.8 & 3.3 & -21.8 & -26.9 \\
\hline Cattle & Lo200 & Louviers & Right humerus & A c12 29,85 & Late Atlantic & 44.5 & 15.2 & 3.4 & -21.8 & -26.9 \\
\hline Cattle & Lo700 & Louviers & Left radius & A c12 54,84 & Late Atlantic & 43.6 & 14.8 & 3.4 & -22.5 & -27.6 \\
\hline Cattle & Lo1300 & Louviers & Left tibia & A c12 35,81 & Late Atlantic & 45.2 & 15.1 & 3.5 & -22.6 & -27.7 \\
\hline Cattle & Lo1400 & Louviers & Right radius & A c12 30,83 & Late Atlantic & 46.2 & 15.8 & 3.4 & -22.1 & -27.2 \\
\hline Cattle & Lo1600 & Louviers & Right radius & A c18 57,79 & Late Atlantic & 43.0 & 14.9 & 3.4 & -23.1 & -28.2 \\
\hline Cattle & Lo1700 & Louviers & Right radius & A c18 22,77 & Late Atlantic & 44.5 & 14.9 & 3.5 & -21.2 & -26.3 \\
\hline Cattle & Lo1900 & Louviers & Left radius & A c18 62,77 & Late Atlantic & 44.5 & 14.8 & 3.5 & -22.4 & -27.5 \\
\hline Cattle & Lo2000 & Louviers & Right tibia & A c18 53,80 & Late Atlantic & 44.4 & 14.7 & 3.5 & -21.8 & -26.9 \\
\hline Cattle & Lo2100 & Louviers & Right tibia & A c18 42,77 & Late Atlantic & 44.5 & 15.2 & 3.4 & -22.3 & -27.4 \\
\hline
\end{tabular}

Tissue $\delta^{13} \mathrm{C}_{\text {coll }}$ values correspond to $\delta^{13} \mathrm{C}$ values measured on skeletal collagen; $\delta^{13} \mathrm{C}_{\text {diet }}$ values correspond to the $\delta^{13} \mathrm{C}_{\text {coll }}$ values $-5.1 \%$. n.g. stands for not given.

compared to those from Barnett (1994) for arctic tundra (Fig. 6). Vascular plants do not exhibit significant differences in their $\delta^{13} \mathrm{C}$ values in closed canopy conditions (e.g., Gebauer and Meyer, 2003). It is therefore not surprising to find no difference in the $\delta^{13} \mathrm{C}$ values of ruminants dwelling under closed canopy forest, even with different dietary preferences. Only lichens exhibit systematically more positive $\delta^{13} \mathrm{C}$ values than coeval vascular plants (e.g., Fizet et al., 1995; Heaton, 1999; Jahren et al., 2003). Indeed, reindeer, which consume high amounts of lichen, had more positive $\delta^{13} \mathrm{C}$ values than coeval ruminants, as illustrated by our results from central Saskatchewan and by other works (e.g., Szepanski et al., 1999; Bocherens, 2000; BenDavid et al., 2001; Urton and Hobson, 2005). However, the $\delta^{13} \mathrm{C}$ values of reindeer were significantly lower when they fed in a closed canopy environment. Therefore, the contrast between open and closed environment is clearly reflected in the $\delta^{13} \mathrm{C}$ values of ruminants, independently of their forage preferences.

Our conclusions seem to contradict those presented by Stevens et al. (2006) based on the carbon isotopic study of five European populations of red deer. In their study, the results did not show any significant differences in $\delta^{13} \mathrm{C}$ values although the studied populations lived under different environmental conditions. Moreover, the $\delta^{13} \mathrm{C}$ values obtained for red deer populations living in open environments were claimed to be similar to those of the red deer from the closed forest of Bialowieza. However, several problems with the study of Stevens et al. (2006) make its conclusions problematic. First, the samples were collected between 1986 and 2002, but no correction for the shift in the $\delta^{13} \mathrm{C}$ values of atmospheric $\mathrm{CO}_{2}$ during this period was performed. As illustrated by Long et al. (2005), such corrections are 


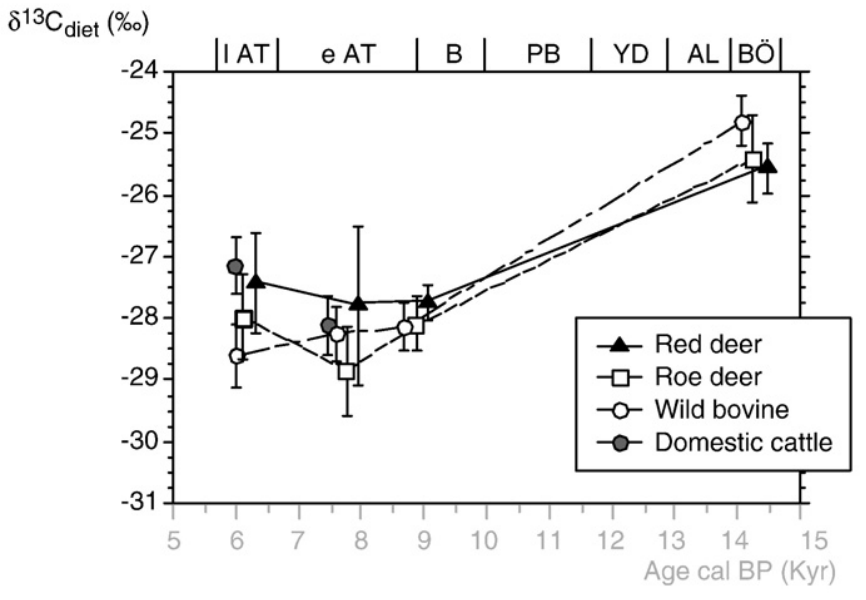

Fig. 4. Mean $\delta^{13} \mathrm{C}_{\mathrm{diet}}$ values of red deer (Cervus elaphus), roe deer (Capreolus capreolus), wild bovine (Bos or Bison) and domestic cattle (Bos taurus) from the Paris Basin during the Late-Glacial/Early Holocene transition. BÖ stands for Bölling, AL stands for Alleröd, YD stands for Younger Dryas, PB stands for Preboreal, B stands for Boreal, e AT stands for early Atlantic and 1 AT stands for late Atlantic.

necessary for reliable ecological studies based on carbon stable isotopes. Moreover, the obtained $\delta^{13} \mathrm{C}$ values were confronted to the $\delta^{13} \mathrm{C}$ values on red deer from Bialowieza Forest published by Bocherens and Drucker (2003), which were not directly comparable to modern data since they were set to Late Pleistocene $\delta^{13} \mathrm{C}$ values of atmospheric $\mathrm{CO}_{2}$. A second problem was the environment of the studied deer populations, for which the degree of closeness of the habitat is loosely described. Moreover, none of these sites correspond to a real dense forest, since the only site considered as "forest environment" was a conifer plantation. Unfortunately, in this type of plantation, ${ }^{13} \mathrm{C}$ depletion in understorey vegetation has been shown to be sometimes lacking due to well-ventilated conditions (e.g., France, 1996; Roche, 1999). One additional point is the fact that Stevens et al. (2006) does not discuss the possible impact of anthropogenic disturbance on red deer ecology in the considered environments. For instance, the deer population from Exmoor is known to cause heavy damage on cultivated crops (e.g. Langbein, 1997; Backshall, 2001; Langbein and Rutter, 2003). In such a context, individuals consuming more crop food than others would exhibit an increase in $\delta^{13} \mathrm{C}$ values since the cultivated plant food growing in open environments present more positive $\delta^{13} \mathrm{C}$ values than plants growing under a forest canopy, which could explain the large range of carbon isotopic values measured in this population.

We therefore still consider that the canopy effect has a significant impact on the $\delta^{13} \mathrm{C}$ values of herbivores dwelling under closed forest conditions, in boreal and temperate contexts.

\subsection{Late-Glacial-Early Holocene transition}

The $\delta^{13} \mathrm{C}_{\text {diet }}$ values of prehistoric large herbivores from Paris Basin, Jura and northern Alps exhibit a decrease of about 3\% during the LateGlacial/Early Holocene transition as previously described in several studies (Drucker et al., 2003; Richards and Hedges, 2003; Stevens and Hedges, 2004; Noe-Nygaard et al., 2005). All these studies agree that the decrease in $\delta^{13} \mathrm{C}$ values of herbivores was clearly related to a decrease in $\delta^{13} \mathrm{C}$ values in the consumed plants. It is therefore crucial to investigate the vegetational changes that occurred during this transition in the studied areas in connection to the observed patterns in herbivore $\delta^{13} \mathrm{C}$ values.

Based on local palaeobotanical and geomorphological studies, the evolution of vegetation in the Paris Basin begins with an increase in arboreal species during the Bölling, but with still a relatively open plant cover and discontinuous woodland (Pastre et al., 2003). After the cold phase of Younger Dryas, the development of deciduous forests starts again during the Preboreal and becomes stable during the Boreal, around 9000 cal BP. These dense forests remain dominant during the early Atlantic and recent Atlantic, despite some local deforestation by Neolithic populations (Pastre et al., 2002). The general evolution of $\delta^{13} \mathrm{C}$ values in Late-Glacial and early Holocene ungulates from the Paris Basin is in keeping with such an evolution of the vegetation cover documented by other approaches, and therefore seem to monitor the increase of the canopy effect during the forest development.

The evolution of vegetation during the Late-Glacial and early Holocene in Jura is documented with great detail, thanks to numerous lake sediment studies (e.g., Peyron et al., 2005; Magny et al., 2006). Shrub vegetation develops at the beginning of the Bölling, followed by the development of open pine-birch woodland. An expansion of open habitats occurs during the cold phase of the Younger Dryas, to the detriment of pine-birch woodland. This woodland environment develops again at the Younger Dryas-Preboreal transition, and meso-thermophilous species, such as hazel, oak and elm, increase in abundance during the Boreal period, when forests of temperate type start to stabilize. Red deer clearly exhibit more positive $\delta^{13} \mathrm{C}$ values during the Late-Glacial periods, when plant cover was shrub

Table 6

Isotopic results for ancient red deer from the Jura

\begin{tabular}{|c|c|c|c|c|c|c|c|c|c|c|c|c|}
\hline Lab $n^{\circ}$ & Site & $\begin{array}{l}\text { Altitude } \\
\text { (m asl) }\end{array}$ & Sample & Excavation $\mathrm{n}^{\circ}$ & Level & $\begin{array}{l}\mathrm{C}_{\text {coll }} \\
(\%)\end{array}$ & $\begin{array}{l}\mathrm{N}_{\text {coll }} \\
(\%)\end{array}$ & $\mathrm{C} / \mathrm{N}_{\text {coll }}$ & $\begin{array}{l}\delta^{13} \mathrm{C}_{\text {coll }} \\
(\%)\end{array}$ & $\begin{array}{l}\delta^{13} C_{\text {diet }} \\
(\%)\end{array}$ & $\begin{array}{l}\text { Age conv. BP } \\
\text { (lab ref) }\end{array}$ & Age cal BP \\
\hline RCD500 & Rochedane & 355 & Left radius & D6 Roch70 n ${ }^{\circ} 19$ & D1 & 42.3 & 15.5 & 3.2 & -19.9 & -25.0 & $12250 \pm 70(\mathrm{GrA21512})$ & $13,902-14,510$ \\
\hline RCD1000 & Rochedane & 355 & Left metatarsal & H9-G9 & $C^{\prime} 1$ & 42.5 & 15.4 & 3.2 & -20.7 & -25.8 & $11,570 \pm 70(\mathrm{GrA21514})$ & $13,270-13,605$ \\
\hline RCD2600 & Rochedane & 355 & Left metatarsal & & B & 38.8 & 14.0 & 3.2 & -20.0 & -25.1 & $11,600 \pm 80(\mathrm{GrA} 21516)$ & $13,282-13,650$ \\
\hline RCD4700 & Rochedane & 355 & Left metatarsal & F9-186 & A4 & 39.7 & 14.8 & 3.1 & -19.5 & -24.6 & $10,880 \pm 50(\mathrm{GrA} 23147)$ & $12,815-12,917$ \\
\hline RCD4800 & Rochedane & 355 & Left metatarsal & H9-190-195 & A4 & 40.7 & 14.7 & 3.2 & -20.6 & -25.7 & $10,830 \pm 70(\mathrm{GrA} 21518)$ & $12,747-12,923$ \\
\hline RCD6300 & Rochedane & 355 & Left metatarsal & $\mathrm{H} 8$ & A3 & 39.1 & 14.3 & 3.2 & -23.4 & -28.5 & $8640 \pm 60(\mathrm{GrA} 21519)$ & 9500-9772 \\
\hline RCD6400 & Rochedane & 355 & Left metatarsal & & A3 & 36.8 & 13.4 & 3.2 & -21.5 & -26.6 & $10,880 \pm 50(\mathrm{GrA} 23150)$ & $12,815-12,917$ \\
\hline RCD10100 & Rochedane & 355 & Left metatarsal & F11-12-135-140 & A2 & 37.4 & 13.5 & 3.2 & -22.8 & -27.9 & $6730 \pm 60($ GrA21520 $)$ & 7490-7679 \\
\hline RCD10500 & Rochedane & 355 & Right metacarpal & Ro72 F10-11 121 & A1 & 42.2 & 16.5 & 3.0 & -23.6 & -28.7 & $6230 \pm 60(\mathrm{GrA} 21522)$ & $6976-7269$ \\
\hline BVN-2 & Bavans & 275 & Right talus & $86 \pi 2$ déc $12 n^{\circ} 13$ & 5 & 37.9 & 13.3 & 3.3 & -23.0 & -28.1 & $6000 \pm 60($ GrA22131) & $6677-6987$ \\
\hline BVN-7 & Bavans & 275 & Metatarsal & O4 déc20 & 7 & 26.1 & 9.8 & 3.1 & -23.4 & -28.5 & $6935 \pm 40($ GrA23127) & 7677-7913 \\
\hline BVN-9 & Bavans & 275 & Jawbone & $\gamma 8-156-160$ no. 5 & terre jaune & 25.9 & 9.6 & 3.2 & -20.8 & -25.9 & $12,170 \pm 60(\mathrm{GrA23129})$ & $13,857-14,171$ \\
\hline RAN-2 & Ranchot & 216 & Left radius & RAN 89 Zg 258 & $\mathrm{AOC}$ & 44.7 & 15.8 & 3.3 & -23.6 & -28.7 & $8570 \pm 60(\mathrm{GrA} 21524)$ & $9470-9674$ \\
\hline RAN-5 & Ranchot & 216 & Left radius & RAN 89 A11 402 & $\mathrm{AOC}$ & 42.7 & 15.2 & 3.3 & -24.0 & -29.1 & $8840 \pm 60(\mathrm{GrA} 21529)$ & 9771-9948 \\
\hline RAN-8(2) & Ranchot & 216 & Left scapula & RAN 83 I11 $125<z<132$ & $\mathrm{AC}$ & 37.3 & 13.9 & 3.1 & -21.9 & -27.0 & $8380 \pm 45(\mathrm{GrA23149})$ & 9289-9491 \\
\hline RAN-9(2) & Ranchot & 216 & Left scapula & RAN 85 G10 112 & $A C$ & 36.8 & 13.6 & 3.1 & -22.9 & -28.0 & $8300 \pm 45(\mathrm{GrA} 25193)$ & $9136-9435$ \\
\hline
\end{tabular}

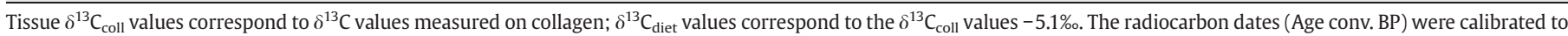
cal years BP using the IntCal04 calibration dataset (Reimer et al., 2004). 
Table 7

Isotopic results for ancient red deer from the Northern Alps

\begin{tabular}{|c|c|c|c|c|c|c|c|c|c|c|c|c|}
\hline $\operatorname{Lab}^{\circ}$ & Site & $\begin{array}{l}\text { Altitude } \\
\text { (m asl) }\end{array}$ & Sample & Excavation $\mathrm{n}^{\circ}$ & Level & $\begin{array}{l}\mathrm{C}_{\text {coll }} \\
(\%)\end{array}$ & $\begin{array}{l}\mathrm{N}_{\text {coll }} \\
(\%)\end{array}$ & $\mathrm{C} / \mathrm{N}_{\text {coll }}$ & $\begin{array}{l}\delta^{13} C_{\text {coll }} \\
(\%)\end{array}$ & $\begin{array}{l}\delta^{13} C_{\text {diet }} \\
(\% \text { ) }\end{array}$ & Age conv. BP & Age cal BP \\
\hline$\overline{\text { FRU-1 }}$ & La Fru & 570 & Metatarsal & Fru III M71 68 & III-2 & 38.1 & 13.7 & 3.2 & -20.1 & -25.2 & $9940 \pm 50(\mathrm{GrA23130})$ & $11,238-11,607$ \\
\hline FRU-2 & La Fru & 570 & Right metacarpal & Fru III K70 14 & III-3 & 35.2 & 13.7 & 3.0 & -22.4 & -27.5 & $8630 \pm 70(\mathrm{GrA} 22134)$ & 9485-9885 \\
\hline FRU-3 & La Fru & 570 & Right metacarpal & Fru III K70 63I & III-4a & 32.7 & 12.4 & 3.1 & -21.1 & -26.2 & $9490 \pm 70(\mathrm{GrA} 22116)$ & $10,574-11,089$ \\
\hline FRU-9 & La Fru & 570 & Right metacarpal & Fru III L70 354 & III-4a & 39.4 & 14.8 & 3.1 & -20.2 & -25.3 & $8630 \pm 70(\mathrm{GrA} 25061)$ & $9485-9885$ \\
\hline FRU-4 & La Fru & 570 & Phalanx I & Fru III L72 591 & III-4c & 29.5 & 11.6 & 3.0 & -20.8 & -25.9 & $11420 \pm 60(\mathrm{GrA} 25062)$ & $13,173-13,401$ \\
\hline FRU-5 & La Fru & 570 & Left talus & Fru III K70 1336 & III-7 & 36.3 & 14.1 & 3.0 & -20.3 & -25.4 & $12260 \pm 90(\mathrm{GrA} 22098)$ & $13,883-14,648$ \\
\hline FRU-10 & La Fru & 570 & Left metacarpal & Fru I F24 129 c1c & $\mathrm{I}-1 \mathrm{c}$ & 43.7 & 16.0 & 3.2 & -19.9 & -25.0 & $11950 \pm 60(\mathrm{GrA} 25052)$ & $13,692-13,964$ \\
\hline FRU-13 & La Fru & 570 & Left metacarpal & Fru I F25 386 c1c & $\mathrm{I}-1 \mathrm{c}$ & 43.3 & 16.0 & 3.2 & -20.2 & -25.3 & $11790 \pm 60(\mathrm{GrA} 25054)$ & $13,465-13,780$ \\
\hline FRU-15 & La Fru & 570 & Left metacarpal & Fru I A9 QAOB FC 7 & $\mathrm{I}-3 ?$ & 42.7 & 15.9 & 3.1 & -21.1 & -26.2 & $11840 \pm 60(\mathrm{GrA25060})$ & $13,536-13,835$ \\
\hline LDT200 & Les Douattes & 370 & Inf P4 & F5-73 & & 41.7 & 15.3 & 3.2 & -20.7 & -25.8 & $12100 \pm 60($ Ly $145300 x A)$ & $13,806-14,095$ \\
\hline LDT600 & Les Douattes & 370 & Metatarsal & D99 F554 & & 41.8 & 15.4 & 3.2 & -19.8 & -24.9 & $12570 \pm 80(\mathrm{GrA} 22132)$ & $14,272-15,085$ \\
\hline APS-2 & Aulp-du-Seuil & 1700 & Right tibia & ALP1 F6 300 & C2 sup & 42.9 & 15.9 & 3.2 & -21.7 & -26.8 & $7110 \pm 50(\mathrm{GrA24775})$ & $7841-8015$ \\
\hline GRV-1 & La Grande Rivoire & 580 & Tibia & 178 & $\mathrm{D}$ & 42.1 & 15.3 & 3.2 & -20.4 & -25.5 & $9160 \pm 50(\mathrm{GrA} 25064)$ & $10,230-10,487$ \\
\hline GRV-5 & La Grande Rivoire & 580 & Phalanx & $2 \mathrm{C}$ & $\mathrm{C}$ & 41.4 & 15.1 & 3.2 & -21.1 & -26.2 & $8640 \pm 50(\mathrm{GrA} 25065)$ & $9528-9731$ \\
\hline GRV-9 & La Grande Rivoire & 580 & Tibia & GRiv-6 55 B3 & B3 & 41.8 & 15.6 & 3.1 & -21.1 & -26.2 & $7435 \pm 45(\mathrm{GrA} 25066)$ & $8178-8357$ \\
\hline
\end{tabular}

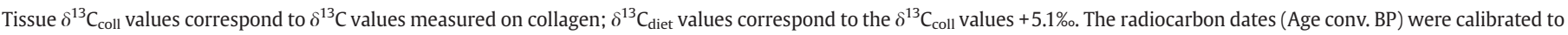
cal years BP using the IntCal04 calibration dataset (Reimer et al., 2004).

vegetation and open woodland, than during the Early Holocene, when vegetation corresponds to dense temperate forest.

Less data are available for the French northern Alps than for Jura, but the evolution of vegetation in the Alps looks broadly similar to that of Jura. The development of forest starts during the Preboreal, with an upper tree limit higher than $2100 \mathrm{~m}$ during the Holocene (Tessier et al., 1993). In contrast to the Jura red deer, those from the French Northern Alps did not exhibit a significant decrease of their $\delta^{13} \mathrm{C}$ values, despite the change of vegetation that occurred below $2000 \mathrm{~m}$. This situation exemplifies the possible role of local parameters, in such case the range of altitudes around the sites corresponding to a range of vegetation type, from dense forests at lower altitudes and more open plant formations at higher altitudes, that can significantly impact the $\delta^{13} \mathrm{C}$ values of large herbivores.

We consider that the canopy effect linked to the increasing forest cover in western Europe is a convincing driving parameter for the observed decline in $\delta^{13} \mathrm{C}$ value of herbivores at the transition between Late-Glacial and Early Holocene. However, besides the canopy effect, more global factors such as the increase in atmospheric $\mathrm{CO}_{2}$ concentration has been suggested as the main cause for the decline in $\delta^{13} \mathrm{C}$ values (Richards and Hedges, 2003; Hedges et al., 2004; Stevens and Hedges, 2004). Their conclusions were inspired by the work of Leavitt and Danzer (1992) who considered increasing

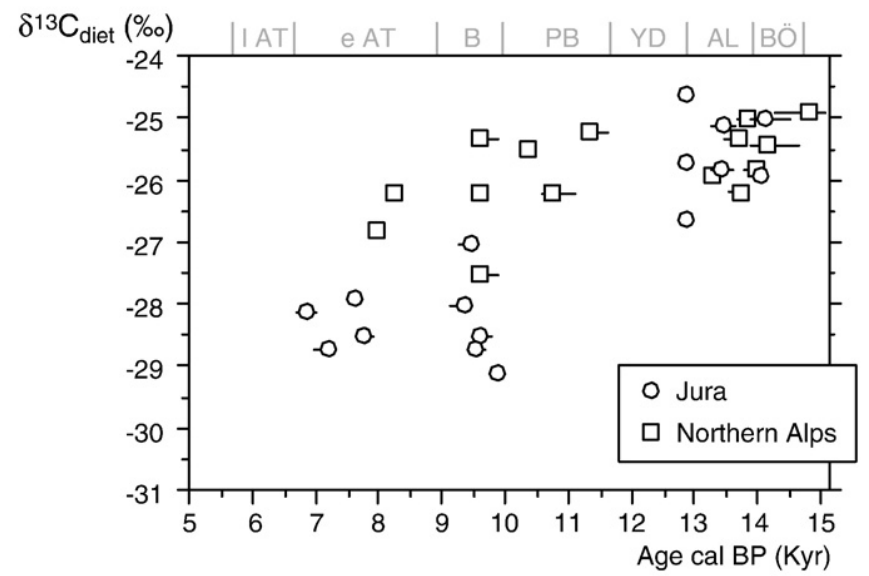

Fig. 5. $\delta^{13} C_{\text {diet }}$ values of red deer (Cervus elaphus) from the Jura and the northern Alps during the Late-Glacial/Early Holocene transition. BÖ stands for Bölling, AL for Alleröd, YD for Younger Dryas, PB for Preboreal, B for Boreal, e AT for early Atlantic and 1 AT for late Atlantic. The horizontal bars correspond to the range of 2 standard-deviations for the calibrated radiocarbon dates if they exceed the size of the circle or square. atmospheric $\mathrm{CO}_{2}$ concentration during the Late-Glacial (Neftel et al., 1988; Smith et al., 1997) as responsible for observed decreasing plant $\delta^{13} \mathrm{C}$ values by 1 to $4 \%$ (Krishnamurthy and Epstein, 1990; Leavitt and Danzer, 1991; van de Water et al., 1994). This hypothesis was supported by experimental results of Poley et al. (1993) who observed such a shift in plants facing an increase in atmospheric $\mathrm{CO}_{2}$ concentration. However, contrary to Poley et al. (1993), experimental work of Arens et al. (2000) concluded that $\delta^{13} \mathrm{C}$ values of plants depended mainly on $\delta{ }^{13} \mathrm{C}$ values of atmospheric $\mathrm{CO}_{2}$. Indeed, plants seem able to adapt their stomatal conductance to variation in atmospheric $\mathrm{CO}_{2}$ concentration and so maintain the same isotopic discrimination against ${ }^{13} \mathrm{C}$ (see Ehleringer and Cerling, 1995). In a context of a constant $\delta^{13} \mathrm{C}$ value of atmospheric $\mathrm{CO}_{2}$, environmental conditions would be primarily influenced by environmental and plant growth-form parameters (Arens et al., 2000). Therefore, increase in atmospheric $\mathrm{CO}_{2}$ is unlikely to be responsible for the observed trend in herbivore $\delta^{13} \mathrm{C}$ values at the Late-Glacial to Early Holocene transition.

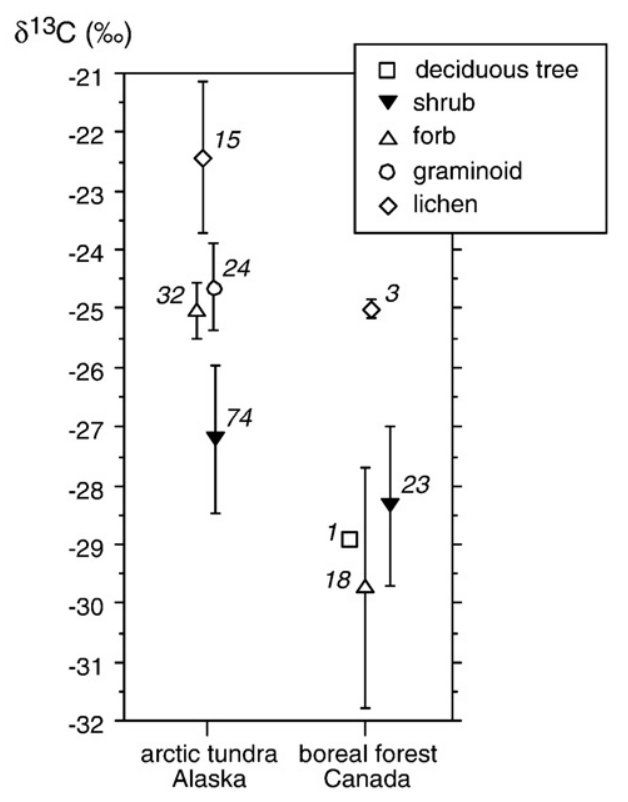

Fig. 6. Comparison of $\delta^{13} \mathrm{C}$ values of plant types in open (tundra: Barnett, 1994) and closed canopy (boreal forest: Brooks et al., 1997) environments. For the boreal forest, only plants growing under 3 meters from the soil are considered since they are the ones reachable to the studied herbivore species. Number of specimens ( $\mathrm{n}$ ) are given. Data for lichen from boreal lichen are unpublished. 
Moreover, there is a chronological discrepancy between the most important increase in atmospheric $\mathrm{CO}_{2}$, that occurs at the end of the Pleistocene and well before the beginning of the Holocene (Smith et al., 1999), and the observed decrease in herbivore $\delta^{13} \mathrm{C}$ values that occur at the beginning of the Holocene.

Also based on a critical reappraisal of the possible impact of atmospheric $\mathrm{CO}_{2}$ on plant $\delta^{13} \mathrm{C}$ values, Hedges et al. (2006) finally consider the drop in $\delta^{13} \mathrm{C}$ values of large mammals at the Late-Glacial to Early Holocene transition as the result of the combination of different environmental and climatic parameters. Among these parameters, forest development was mentioned as a minor component, principally because changes in faunal collagen $\delta^{13} \mathrm{C}$ values were considered to be similar among species and between geographical regions. However, the isotopic data that we present in this paper contradict this pattern.

Indeed, the depletion in $\delta^{13} \mathrm{C}_{\text {diet }}$ values we observed in Paris Basin during the Late-Glacial/Early Holocene transition (ca. 15,000-6000 yr cal BP) differed among species. Differences in $\delta^{13} \mathrm{C}_{\text {diet }}$ values decline pattern among wild bovine, red and roe deer was probably due to species-related change in diet and adaptation to a changing environment. Domestic cattle and wild bovine have the same digestive physiology and, indeed, exhibited comparable $\delta^{13} \mathrm{C}_{\text {diet }}$ values during the early Atlantic. In the late Atlantic, however, cattle $\delta^{13} \mathrm{C}_{\text {diet }}$ values increased toward higher values than those of wild bovine. If we consider the depleted $\delta^{13} \mathrm{C}_{\text {diet }}$ values of wild bovine as the result of under canopy feeding, this could indicate an access to open areas for cattle bred by Middle Neolithic humans. The same pattern of $\delta^{13} \mathrm{C}$ values being less depleted in late Atlantic domestic cattle than in contemporaneous aurochs was observed in Denmark by Noe-Nygaard et al. (2005) and in the French Jura by Bocherens et al. (in press). This pattern in decline of $\delta^{13} \mathrm{C}$ values depending on the considered species suggests that it is due not only to dietary preferences, but also to niche partitioning involving differences in habitat (Stewart et al., 2003).

Finally, red deer from Jura and northern Alps confirm that local differences in patterns of change in $\delta^{13} C_{\text {diet }}$ values of a single species occurred during the Late-Glacial/Early Holocene transition. We interpret this isotopic difference as the result of differences in the extent of forest canopy development in the red deer habitat over time and space. In Jura, red deer $\delta^{13} C_{\text {diet }}$ values, were more depleted after the Younger Dryas than before. This decrease in red deer $\delta^{13} \mathrm{C}_{\text {diet }}$ values over time was consistent with the development of dense deciduous forests composed of hazelnut (Corylus), oak (Quercus) and elm (Ulmus) at the end of the Preboreal as shown by pollen analysis (Richard et al., 2000). The northern Alps area yielded higher $\delta^{13} C_{\text {diet }}$ values through the Late-Glacial/Early Holocene transition. This can be explained by the presence of habitat more exposed in this region than in Jura, probably linked to the high altitude of the sites (from 370 to $1700 \mathrm{~m}$ ) and their surroundings. Although $\delta^{13} \mathrm{C}$ values of plants have been shown to increase with altitude at a rate of $0.7 \%$ / 1000 m (Körner et al., 1988). Thus, a difference in altitude of more than 3000 meters would be required to account for the observed difference in red deer $\delta^{13} \mathrm{C}$ values between Jura and northern Alps, all other parameters being equal. The mere altitude difference is therefore not sufficient to explain the observed trend. A much more likely explanation is the environmental contrast between the studied sites of Jura and those from the Alps. Indeed, the sites of Jura are located in the Doubs valley less than $360 \mathrm{~m}$ asl and are connected largely with adjacent plains where the dense canopy forest could have spread more rapidly than in the Northern Alps sites where red deer were hunted.

\section{Conclusion}

The study of well-monitored free-ranging populations of cervids and bovines confirms that the carbon isotope signatures of ruminants can reflect the canopy effect. This allows, in pure $C_{3}$ environments, to distinguish herbivore populations feeding under closed canopy forests from populations feeding in more open environments. It is important to bear in mind that the so called "canopy effect" results from the convergent effects of particular conditions, involving abiotic factors, such as temperature, humidity, and air circulation, as well as biotic factors, such as the floristic composition, growth stage and canopy complexity. Not all woodland types will generate a canopy effect. However, when a canopy effect is observed, it implies the occurrence of mature, dense and complex forest stands. Having a natural tracker of the development of such environments, the carbon isotope composition of large herbivore tissues, allows to complement the other palaeoenvironmental tracers, especially palaeobotanical ones. In ancient contexts of forest expansion, such as the Late-Glacial to Early Holocene transition, it offers a means to document the habitat shift of herbivores hunted by prehistoric populations, and to compare patterns of environmental change during this period of global warming.

\section{Acknowledgements}

We thank those who provided us with samples and scientific support : Céline Bemilli (INRAP, UMR 5197), Pierre Bodu (UMR 7041 Nanterre), André Cholet and Véronique Dujardin (DRAC PoitouCharentes, Poitiers), Christophe Cupillard (SRA France-Comté, Besançon), Anne Gunn (Ressources, Wildlfe and Economic Development, Yellowknife); François Giligny (UMR 7041), Christophe Griggo (Institut Dolomieu, Grenoble), Louis Chaix (Museum d'Histoire Naturelle, Genève), Yves Lanchon (INRAP, UMR 7041), Gilbert Pion (UMR 6565, Besançon), Gustaf Samelius (CWS, Saskatoon), Pr. André Thévenin (Besançon), Anne Tresset, Jean-Denis Vigne (MNHN, UMR 5197). Sample preparation and isotopic measurements were conducted with the technical assistance of Daniel Billiou (UMR 7618), Patricia Healy (CWS, Saskatoon) and Issam Moussa (UMR 5554). We thank the Department of Soil Science, University of Saskatchewan, for the isotopic analysis of caribou hair. We would also like to thank Bruce McFadden for his useful comments on an earlier version of this manuscript. Financial support from the ECLIPSE "Cervus" program of CNRS, French PCR program "La fin du Paléolithique supérieur dans les Alpes du Nord françaises et le Jura méridional” and Fyssen foundation is gratefully acknowledged.

\section{References}

Aaris-Sorensen, K., Mühldorff, R., Petersen, E.B., 2007. The Scandinavian reindeer (Rangifer tarandus L.) after the last glacial maximum: time, seasonality and human exploitation. J. Archaeol. Sci. 34, 914-923.

Ambrose, S.H., 1990. Preparation and characterization of bone and tooth collagen for isotopic analysis. J. Archaeol. Sci. 17, 431-451.

Ambrose, S.H., 1993. Isotopic analysis of paleodiets: methodological and interpretive considerations. In: Standford, M.K. (Ed.), Investigations of ancient human tissue, chemical analyses in anthropology. Gordon and Breach Science Publishers, Langhorne, pp. 59-130.

Ambrose, S.H., DeNiro, M.J., 1986. The isotopic ecology of East African mammals Oecologia 69, 395-406.

Amman, B., Lotter, A.F., 1989. Late-Glacial radiocarbon- and palynostratigraphy on the Swiss Plateau. Boreas 18, 109-126.

Arens, N.C., Jahren, A.H., Amundson, R., 2000. Can C 3 plants faithfully record the carbon isotopic composition of atmospheric carbon dioxide? Paleobiology 26, 137-164.

Ayliffe, L.K., Cerling, T.E., Robinson, T., West, A.G., Sponheimer, M., Passey, B.H., Hammer J., Roeder, B., Dearing, M.D., Ehleringer, J.R., 2004. Turnover of carbon isotopes in tail hair and breath $\mathrm{CO}_{2}$ of horses fed an isotopically varied diet. Oecologia 139, 11-22.

Baales, M., 1996. Umwelt und Jagdökonomie der Ahrensburger Rentierjäger im Mittlegebirge. Monographien Römisch-Germanisches Zentralmuseum, 38. Habelt, Bonn. 364 pp.

Backshall, J., 2001. Moorland. In: Backshall, J., Manley, J., Rebane, M. (Eds.), The Upland management handbook. English Nature, Peterborough, UK, p. 6:1-6:130.

Balasse, M., 1999. De l'exploitation du lait au Néolithique moyen, en Europe tempérée Examen des modalités de sevrage des bovins, par l'analyse isotopique des ossements archéologiques. Ph.D. Thesis, University of Paris 6, Paris, 169 pp.

Barnett, B.A. 1994. Carbon and nitrogen isotope ratios of caribou tissues, vascular plants and lichens from Northern Alaska. M.Sc. Thesis, University of Alaska, Fairbanks, Alaska, USA

Beaulieu, J.-L., de Andrieu, V., Ponel, P., Reille, M., Lowe, J.J., 1994a. The Weichselian LateGlacial in southwestern Europe (Iberian Peninsula, Pyrenees, Massif Central, northern Apennines). J. Quat. Sci. 9, 101-107. 
Beaulieu, J.-L., de Richard, H., Ruffaldi, P., Clerc, J., 1994b. History of vegetation, climate and human action in the French Alps and the Jura over the last 15,000 years. Dissertationes Botanicae 234, 253-275.

Ben-David, M., Shochat, E., Adams, L.G., 2001. Utility of stable isotope analysis in studying foraging ecology of herbivores: examples from moose and caribou. Alces 37, 421-434.

Bocherens, H., 2000. Preservation of isotopic signals $\left({ }^{13} \mathrm{C},{ }^{15} \mathrm{~N}\right)$ in Pleistocene mammals In: Katzenberg, M.A., Ambrose, S.H. (Eds.), Biogeochemical approaches to Paleodietary Analyses. Kluwer Academic/Plenum Publishers, New York, pp. 65-88.

Bocherens, H., Drucker, D., 2003. Trophic level isotopic enrichments for carbon and nitrogen in collagen: case studies from recent and ancient terrestrial ecosystems. International Journal of Osteoarchaeology 13, 46-53.

Bocherens, H., Pacaud, G., Lazarev, P., Mariotti, A., 1996. Stable isotope abundances $\left({ }^{13} \mathrm{C}\right.$, ${ }^{15} \mathrm{~N}$ ) in collagen and soft tissues from Pleistocene mammals from Yakutia. Implications for the paleobiology of the mammoth steppe. Palaeogeogr., Palaeoclimatol., Palaeoecol. 126, 31-44.

Bocherens, H., Billiou, D., Patou-Mathis, M., Bonjean, D., Otte, M., Mariotti, A., 1997. Paleobiological implications of the isotopic signature $\left({ }^{13} \mathrm{C},{ }^{15} \mathrm{~N}\right)$ of fossil mammal collagen in Scladina cave (Sclayn, Belgium). Quatern. Res. 48, 370-380

Bocherens, H., Billiou, D., Tresset, A., 2005a. Approche biogéochimique $\left({ }^{13} \mathrm{C},{ }^{15} \mathrm{~N}\right) \mathrm{de}$ l'exploitation de l'environnement par les humains. In: Giligny, F. (Ed.), Louviers "La Villette" (Eure): un site néolithique moyen en zone humide. Documents Archéologies de l'Ouest, Rennes, pp. 265-269.

Bocherens, H., Drucker, D., Billiou, D., Patou-Mathis, M., Vandermeersch, B., 2005b. Isotopic evidence for diet and subsistence pattern of the Saint-Césaire I Neanderthal: review and use of a multi-source mixing model. J. Hum. Evol. 49, 71-87.

Bocherens, H., Arbogast, R., Billiou, D., Drucker, D., Herrscher, E., in press. Biogéochimie isotopique des ossements animaux : contribution à la reconstitution des paléomilieux et de leur utilisation par les populations humaines. In: Pétrequin, P., Pétrequin, A.-M. (Eds.), Les sites littoraux néolithiques de Clairvaux et de Chalain (Jura), tome IV : du Ferrières au groupe de Clairvaux ( $31^{\mathrm{e}}$ et $30^{\mathrm{e}}$ siècles av. J.-C.).

Bridault, A., Chaix, L., 2002. Ruptures et équilibres dans les faunes à la fin du Pléistocène et durant l'Holocène ancien en Europe occidentale. In: Richard, H., Vignot, A. (Eds.), Equilibres et ruptures dans les écosystèmes durant les 20 derniers millénaires en Europe de l'Ouest. Actes du colloque international de Besançon. Presses universitaires Franc-Comtoises, Besançon, pp. 53-60.

Bridault, A., Chaix, L., Pion, G., Oberlin, C., Thiébault, S., Argant, J., 2000. Position chronologique du renne (Rangifer tarandus L.) à la fin du Tardiglaciaire dans les Alpes du Nord françaises et le Jura méridional. Mém. Soc. Préhist. Fr. 28, 47-57.

Broadmeadow, M.S.J., Griffiths, H., 1993. Carbon isotope discrimination and the coupling of $\mathrm{CO}_{2}$ fluxes within forest canopies. In: Ehleringer, J.R., Hall, A.E. Farquhar, G.D. (Eds.), Stable isotopes and plant carbon-water relations. Academic Press, Inc., San Diego, pp. 109-129.

Broadmeadow, M.S.J., Griffiths, H., Maxwell, C., Borland, A.M., 1992. The carbon isotope ratio of plant organic material reflects temporal and spatial variations in $\mathrm{CO}_{2}$ within tropical forest formations in Trinidad. Oecologia 89, 435-441.

Brooks, J.R., Flanagan, L.B., Buchmann, N., Ehleringer, J.R., 1997. Carbon isotope composition of boreal plants: functional grouping of life forms. Oecologia 110, 301-311.

Buchmann, N., Wen-Yuan, K., Ehleringer, J.R., 1997. Influence of stand structure on carbon-13 of vegetation, soils, and canopy air within deciduous and evergreen forests in Utah, United States. Oecologia 110, 109-119.

Cerling, T.E., Harris, J.M., MacFadden, B.J., 1998. Carbon isotopes, diets of North American Equids, and the evolution of North American $\mathrm{C}_{4}$ grasslands. In: Griffith, H., Robinson, D., van Gardingen, R.P. (Eds.), Stable isotopes and integration of biological, ecological and geochemical processes. Bios Scientific, Oxford, pp. 363-379.

Charles, R., 1993. Evidence for faunal exploitation during the Belgian Lateglacial: recent research on the Dupont collection from the Trou de Chaleux. In: Desse, J., AudouinRouzeau, F. (Eds.), Exploitation des animaux sauvages à travers le temps. XIIèmes rencontres internationales d'Archéologie et d'Histoire d'Antibes. APDCA, Juan-LesPins, pp. 103-115.

Coard, R., Chamberlain, A.T., 1999. The nature and timing of faunal change in the British Isles across the Pleistocene/Holocene transition. The Holocene 9, 372-376.

Coppedge, B.R., Leslie Jr., D.M., Shaw, J.H., 1998. Botanical composition of bison diets on tallgrass prairie in Oklhahoma. J. Range Manage. 51, 379-382.

Dawson, T.E., Mambelli, S., Plamboeck, A.H., Templer, P.H., Tu, K.P., 2002. Stable isotopes in plant ecology. Annu. Rev. Ecol. Syst. 33, 507-559.

de Bellefeuille, S., 2001. Le caribou forestier et la sylviculture. Ministère des ressources naturelles, Québec. $91 \mathrm{pp}$

Drucker, D., Bocherens, H., Pike-Tay, A., Mariotti, A., 2001. Isotopic tracking of seasonal dietary change in dentine collagen: preliminary data from modern caribou. C.R. Acad. Sci., Paris 333 II a, 303-309.

Drucker, D., Bocherens, H., Bridault, A., Billiou, D., 2003. Carbon and nitrogen isotopic composition of red deer (Cervus elaphus) collagen as a tool for tracking palaeoenvironmental change during the Late-Glacial and Early Holocene in the northern Jura (France). Palaeogeogr. Palaeoclimatol. Palaeoecol. 195, 375-388.

Ehleringer, J.R., Cerling, T.E., 1995. Atmospheric $\mathrm{CO}_{2}$ and the ratio of intercellular to ambient $\mathrm{CO}_{2}$ concentrations in plants. Tree Physiology 15, 105-111.

Ehleringer, J.R., Field, C.B., Lin, Z.F., Kuo, C.Y., 1986. Leaf carbon isotope and mineral composition in subtropical plants along an irradiance cline. Oecologia 70, 520-526.

Feng, X 1998. Long-term $c_{i} / c_{a}$ response of trees in western North America to atmospheric $\mathrm{CO}_{2}$ concentration derived from carbon isotope chronologies. Oecologia 117, 19-25.

Fizet, M., Mariotti, A., Bocherens, H., Lange-Badré, B., Vandermeersch, B., Borel, J.P. Bellon, G., 1995. Effect of diet, physiology and climate on carbon and nitrogen isotopes of collagen in a late Pleistocene anthropic paleoecosystem (France, Charente, Marillac). J. Archaeol. Sci. 22, 67-79.

Flerov, K.K., 1952. Musk Deer and Deer. Izdatelstvo Akademii Nauk SSSR, Moscow.

Fortin, D., Fryxell, J.M., O'Brodovich, L., Frandsen, D., 2003. Foraging ecology of bison at the landscape and plant community levels: the applicability of energy maximization principles. Oecologia 134, 219-227.

France, R., 1996. Carbon isotope ratios in logged and unlogged boreal forests examination of the potential for determining wildlife habitat use. Environmental Management 20, 249-255.

Francey, R.J., Gifford, R.M., Sharkey, T.D., Weir, B., 1985. Physiological influences on carbon isotope discrimination in huon pine (Lagarostrobos franklinii). Oecologia 66, 211-218.

Gebauer, G., Meyer, M., 2003. ${ }^{15} \mathrm{~N}$ and ${ }^{13} \mathrm{C}$ natural abundance of autotrophic and mycoheterotrophic orchids provides insight into nitrogen and carbon gain from fungal association. New Phytologist 160, 209-223.

Gebauer, G., Schulze, E.-D., 1991. Carbon and nitrogen isotope ratios in different compartments of a healthy and a declining Picea abies forest in the Fichtelgebirge, NE Bavaria. Oecologia 87, 198-207.

Gebczynska, Z., 1980. Food of the roe deer and red deer in the Bialowieza Primeval Forest. Acta Theriologica 25, 487-500.

Gebert, C., Verheyden-Tixier, H., 2001. Variations of diet composition of Red Deer (Cervus elaphus L.) in Europe. Mammal Rev. 31, 189-201.

Geist, V., 1999. Deer of the world. Swan Hill Press, Shrewsbury, England. 421 pp.

Heaton, T.H.E., 1999. Spatial, species, and temporal variations in the ${ }^{13} \mathrm{C} /{ }^{12} \mathrm{C}$ ratios of $\mathrm{C}_{3}$ plants: implications for palaeodiet studies. J. Archaeol. Sci. 26, 637-649.

Hedges, R.E.M., Stevens, R.E., Richards, M.P., 2004. Bone as stable isotope archive for local climatic information. Quat. Sci. Rev. 23, 959-965.

Hedges, R.E.M., Stevens, R.E., Koch, P.L., 2006. Isotopes in bones and teeth. In: Leng, M.J. (Ed.), Isotopes in palaeoenvironmental research, Developments in palaeoenvironmental research, 10, pp. 117-145.

Heptner, V.G., Nasimovitch, A.A., Bannikov, A.G., 1989. Mammals of the Soviet Union, Vol.1 Ungulates. E.J. Brill, Leiden.

Hoffmann, R.R.,1989. Evolutionary steps of ecophysiological adaptation and diversification of ruminants: a comparative view of their digestive system. Oecologia 78, 443-457.

Hufthammer, A.K., Aaris-Sorensen, K., 1998. Late- and postglacial European roe deer. In: Anderson, R., Duncan, P., Linnell, J.D.C. (Eds.), The European roe deer: the biology of success. Scandinavian University Press, pp. 47-69.

Jahren, A.H., Porter, S., Kuglitsch, J.J., 2003. Lichen metabolism identified in Early Devonian terrestrial organisms. Geology 31, 99-102.

Kelsall, J.P., 1968. The migratory barren-ground caribou of Canada. Canadian Wildlife Service, Ottawa. 339 pp.

Kie, J.G., Bowyer, R.T., Stewart, K.M., 2003. Ungulates in western forests: habitat requirements, population dynamics, and ecosystem processes. In: Zabel, C.J., Anthony, R.G. (Eds.), Mammal community dynamics: management and conservation in the coniferous forests of western North America. Cambridge University Press, Cambridge, UK, pp. 296-340.

Körner, C., Farquhar, G.D., Roksandic, Z., 1988. A global survey of carbon isotope discrimination in plants from high altitude. Oecologia 74, 623-632.

Krishnamurthy, R.V., Epstein, S., 1990. Glacial-Interglacial excursion in the concentration of atmospheric $\mathrm{CO}_{2}$-effect in the ${ }^{13} \mathrm{C} /{ }^{12} \mathrm{C}$ ratio in wood cellulose. Tellus $\mathrm{B}$ Chem. Phys. Meteorol. 42, 423-434.

Langbein, J., 1997. The ranging behaviour, habitat-use and impact of deer in oak woods and heather moors of Exmoor and the Quantock Hills. British Deer Society, Fordingbridge.

Langbein, J., Rutter, S.M., 2003. Quantifying the damage wild deer cause to agricultural crops and pastures. In: Goldberg, E. (Ed.), Proceedings of future for deer conference 28 \& 29 March 2003. English Nature Research Reports, vol. 548, pp. 32-39.

Leavitt, S.W., Danzer, S.R., 1991. Chronology from plant matter. Nature 352, 671.

Leavitt, S.W., Danzer, S.R., 1992. $\delta^{13} \mathrm{C}$ variations in $C_{3}$ plants over the past 50,000 years. Radiocarbon 34, 783-791.

Leuenberger, M., Siegenthaler, U., Langway, C.C., 1992. Carbon isotope composition of atmospheric $\mathrm{CO}_{2}$ during the last ice age from an Antarctic ice core. Nature 357, 488-490.

Lindgren, E., Pulliainen, E., Sulkava, S., Erkinaro, E., Heikura, K., 1983. Lichen resources and their use in winter by wild forest reindeer in the area of Lake Lentua. Acta Zoologica Fennica 175, 21-23.

Linnell, J.D.C., Duncan, P., Andersen, R., 1998. The European roe deer: a portrait of a successful species. In: Anderson, R., Duncan, P., Linnell, J.D.C. (Eds.), The European roe deer: the biology of success. Scandinavian University Press, Oslo, pp. 11-22.

Long, E.S., Sweitzer, R.A., Diefenbach, D.R., Ben-David, M., 2005. Controlling for anthropogenically induced atmospheric variation in stable carbon isotope studies. Oecologia 146, 148-156.

Magny, M., Aalbersberg, G., Bégeot, C., Benoit-Ruffaldi, P., Bossuet, G., Disnar, J.R., Heiri, O., Laggoun-Defarge, F., Mazier, F., Millet, L., Peyron, O., Vannière, B., WalterSimmonnet, A.V., 2006. Environmental and climatic changes in the Jura mountains (eastern France) during the Late Glacial-Holocene transition: a multi-proxy record from Lake Lautrey. Quat. Sci. Rev. 25, 414-445.

Mangerud, J., Andersen, S.T., Berglund, B.E., Donner, J.J., 1974. Quaternary stratigraphy of Norden, a proposal for terminology and classification. Boreas 3, 109-128.

Neftel, A., Oeschger, H., Staffelbach, T., Stauffer, B., 1988. $\mathrm{CO}_{2}$ record in the Byrd ice core 50,000-5000 years BP. Nature 331, 609-611.

Noe-Nygaard, N., Price, T.D., Hede, S.U., 2005. Diet of aurochs and early cattle in southern Scandinavia: evidence from ${ }^{15} \mathrm{~N}$ and ${ }^{13} \mathrm{C}$ stable isotopes. J. Archaeol. Sci. 32, 855-871.

Pastre, J.F., Leroyer, C., Limondin-Lozouet, N., Orth, P., Chaussé, C., Fontugne, M., Gauthier, A., Kunesch, S., Le Jeune, Y., Saad, M.C., 2002. Variations paléoenvironnementales et paléohydrologiques durant les 15 derniers millénaires: les 
réponses morphosédimentaires des vallées du Bassin Parisien (France). In: Bravard, J.P., Magny, M. (Eds.), Les fleuves ont une histoire. Errance, St Etienne, pp. 29-44.

Pastre, J.F., Leroyer, C., Limondin-Lozouet, N., Antoine, P., Gauthier, A., Le Jeune, Y., Orth, P., 2003. Quinze mille ans d'environnement dans le Bassin Parisien (France): mémoires sédimentaires des fonds de vallée. In: Muxart, T., Vivien, F.-D., Villalba, B., Burnouf, J. (Eds.), Des milieux et des hommes: fragments d'histoires croisées. Elsevier SAS collection "Environnement”, Paris, pp. 43-55.

Peyron, O., Bégeot, C., Brewer, S., Heiri, O., Magny, M., Millet, L., Ruffaldi, P., Van Campo, E., Yu, G., 2005. Late-glacial climatic changes in Eastern France (Lake Lautrey) from pollen, lake-levels, and chironomids. Quatern. Res. 64, 197-211.

Plumb, G.E., Dodd, J.L., 1993. Foraging ecology of Bison and Cattle on a mixed prairie: Implications for natural area management. Ecological Applications 3, 631-643.

Poley, H.W., Johnson, H.B., Marino, B.D., Mayeux, H.S., 1993. Increase in C 3 plant wateruse efficiency and biomass over Glacial to present $\mathrm{CO}_{2}$ concentrations. Nature 361, 61-64.

Pucek, Z., Belousova, I.P., Krasinska, M., Krasinski, Z.A., Olech, W., 2002. European bison Bison bonasus: current state of the species and an action for its conservation. Mammal Research Institute, Polish Academy of Sciences, Bialowieza, Poland.

Reimer, P.J., Baillie, M.G.L., Bard, E., Bayliss, A., Beck, J.W., Bertrand, C.J.H., Blackwell, P.G., Buck, C.E., Burr, G.S., Cutler, K.B., Damon, P.E., Edwards, R.L., Fairbanks, R.G., Friedrich, M., Guilderson, T.P., Hogg, A.G., Hughen, K.A., Kromer, B., McCormac, F.G., Manning, S., Bronk Ramsey, C., Reimer, R.W., Remmele, R.S., Southon, J.R., Stuiver, M., Talamo, S., Taylor, F.W., van der Plicht, J., Weyhenmeyer, C.E., 2004. INTCAL04 terrestrial radiocarbon age calibration, 0-26 cal kyr BP. Radiocarbon 46, 1029-1058.

Rettie, W.J., Sheard, J.W., Messier, F., 1997. Identification and description of forested vegetation communities available to woodland caribou: relating wildlife habitat to forest cover data. Forest Ecology and Management 93, 245-260.

Richard, H., Bégeot, C., Gauthier, E., Ruffaldi, P., 2000. L'évolution du couvert végétal. In: Cupillard, C., Richard, A. (Eds.), Les derniers chasseurs-cueilleurs du Massif Jurassien et de ses marges (13000-5500 avant Jésus-Christ). Presses Universitaires Franc-Comtoises, Besançon, pp. 29-36.

Richards, M.P., Hedges, R.E.M., 2003. Variations in bone collagen $\delta^{13} \mathrm{C}$ and $\delta^{15} \mathrm{~N}$ values of fauna from Northwest Europe over the last 40000 years. Palaeogeogr. Palaeoclimatol. Palaeoecol. 193, 261-267.

Roche, C., 1999. Transfert de masse, de chaleur et d'isotope dans le continuum solplante-atmosphère : les couverts forestiers. Ph.D. Thesis, University of Paris 6, Paris.

Rodière, E., Bocherens, H., Angibault, J.-M., Mariotti, A., 1996. Particularités de l'azote chez le chevreuil (Capreolus capreolus L.): implications pour les reconstitutions paléoenvironnementales. C.R. Acad. Sci., Paris 323 Ila, 179-185.

Russell, H.J., 1998. The nature of caribou: Spirit of the North. Greystone Books, Vancouver. $114 \mathrm{pp}$.

Schleser, G.H., Jayasekera, R., 1985. $\delta^{13} \mathrm{C}$-variations of leaves in forests as an indication of reassimilated $\mathrm{CO}_{2}$ from the soil. Oecologia $65,536-542$.

Schoeninger, M.J., Iwaniec, U.T., Glander, K.E., 1997. Stable isotope ratios indicate diet and habitat use in New World monkeys. Am. J. phys. Anthrop. 103, 69-83.

Smith, H.J., Wahlen, M., Mastroianni, D., Taylor, K.C., 1997. The $\mathrm{CO}_{2}$ concentration of air trapped in GISP2 ice from the Last Glacial Maximum-Holocene transition. Geophys. Res. Lett. 24, 1-4.

Smith, H.J., Fisher, H., Wahlen, M., Mastroianni, D., Deck, B., 1999. Dual modes of the carbon cycle since the Last Glacial Maximum. Nature 400, 248-250.

Sponheimer, M., Robinson, T., Ayliffe, L., Passey, B., Roeder, B., Shipley, L., Lopez, E., Cerling, T., Dearing, D., Ehleringer, J., 2003. An experimental study of carbon-isotope fractionation between diet, hair, and feces of mammalian herbivores. Can. J. Zool. 81, 871-876.

Stevens, R.E., Hedges, R.E.M., 2004. Carbon and nitrogen stable isotope analysis of northwest European horse bone and tooth collagen, 40,000 BP-present: Palaeoclimatic interpretations. Quat. Sci. Rev. 23, 977-991.

Stevens, R.E., Lister, A.M., Hedges, R.E.M., 2006. Predicting diet, trophic level and palaeoecology from bone stable isotope analysis: a comparative study of five red deer populations. Oecologia 149, 12-21.

Stewart, K.M., Bowyer, R.T., Kie, J.G., Dick, B.L., Ben-David, M., 2003. Niche partitioning among mule deer, elk, and cattle: do stable isotopes reflect dietary niche? Ecoscience 10, 297-302.

Szepanski, M.M., Ben-David, M., Van Ballenberghe, V., 1999. Assessment of anadromous salmon resources in the diet of the Alexander Archipelago wolf using stable isotope analysis. Oecologia 120, 327-335.

Tessier, L, de Beaulieu, J.L. Couteaux, M., Edouard, J.L, Ponel, P., Rolando, C. Thinon, M. Thomas, A., Tobolski, K., 1993. Holocene palaeoenvironments at the timberline in the French Alps - a multidisciplinary approach. Boreas 22, 244-254.

Tieszen, L.L., 1991. Natural variations in the carbon isotope values of plants: implications for archaeology, ecology, and paleoecology. J. Archaeol. Sci. 18, 227-248.

Ukkonen, P., Lougas, L., Zagorska, I., Luksevica, L., Luksevics, E., Daungnora, L., Jungner H., 2006. History of the reindeer (Rangifer tarandus) in the eastern Baltic region and its implications for the origin and immigration routes of the recent northern European wild reindeer populations. Boreas 35, 222-230.

Urton, E.J.M., Hobson, K.A., 2005. Intrapopulation variation in gray wolf isotope $\left(\delta^{15} \mathrm{~N}\right.$ and $\delta^{13} \mathrm{C}$ ) profiles: implications for the ecology of individuals. Oecologia 145, 316-325.

van der Merwe, NJ. 1989. Natural variation in ${ }^{13} \mathrm{C}$ concentration and its effect on environmental reconstruction using ${ }^{13} \mathrm{C} /{ }^{12} \mathrm{C}$ ratios in animal bones. In: Price, T.D. (Ed.), The chemistry of prehistoric human bone. Cambridge University Press, Cambridge, pp. 105-125.

van der Merwe, N.J., Lee-Thorp, J.A., Thackeray, J.F., Hall-Martin, A., Kruger, F.J., Coetzee H., Bell, R.H.V., Lindeque, M., 1990. Source-area determination of elephant ivory by isotopic analysis. Nature 346, 744-746.

van der Merwe, N.J., Medina, E., 1991. The canopy effect, carbon isotope ratios and foodwebs in Amazonia. J. Archaeol. Sci. 18, 249-259.

van de Water, P.K., Leavitt, S.W., Betancourt, J.L., 1994. Trends in stomatal density and ${ }^{13} \mathrm{C} /{ }^{12} \mathrm{C}$ ratios of Pinus flexilis needles during last Glacial-Interglacial cycle. Science 264, 239-243.

Vogel, J.C., 1978. Isotopic assessment of the dietary habits of ungulates, S. Afr. J. Sci. 74 298-301.

Vogel, J.C., Eglington, B., Auret, J.M., 1990. Isotope fingerprints in elephant bone and ivory. Nature 346, 747-749.

von Koenigswald, W., 1999. Palökologie und Vorkommen des pleistozänen Auerochsen (Bos primigenius Bojanus, 1827) im vergleich zu den grossen Rindern des Pleistozäns. In: Weniger, G.C. (Ed.), Archaeology and biology of the aurochs. Wissenschaftliche Schriften, vol. 1. Neanderthal Museum, pp. 23-33.

Weber, A.W., Link, D.W., Katzenberg, M.A., 2002. Hunter-gatherer culture change and continuity in the Middle Holocene of the Cis-Baikal, Siberia. J. Anthrop. Archaeol. 21, 230-299. 